DESY $12-114$

\title{
Confronting the MSSM and the NMSSM with the Discovery of a Signal in the two Photon Channel at the LHC
}

\author{
R. Benbrik牶, M. Gomez Bock讯, S. Heinemeyer㕪,

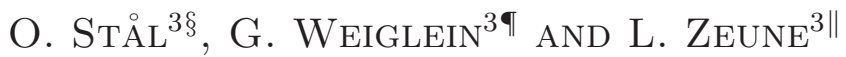 \\ ${ }^{1}$ Instituto de Física de Cantabria (CSIC-UC), Santander, Spain \\ ${ }^{2}$ Facultad de Ciencias Físico-Matemáticas Benemérita Universidad Autónoma de Puebla, \\ Pue. México \\ ${ }^{3}$ DESY, Notkestraße 85, D-22607 Hamburg, Germany
}

\begin{abstract}
We confront the discovery of a boson decaying to two photons, as reported recently by ATLAS and CMS, with the corresponding predictions in the Minimal Supersymmetric Standard Model (MSSM) and the Next-to-Minimal Supersymmetric Standard Model (NMSSM). We perform a scan over the relevant regions of parameter space in both models and evaluate the MSSM and NMSSM predictions for the dominant Higgs production channel and the photon photon decay channel. Taking into account the experimental constraints from previous direct searches, flavour physics, electroweak measurements as well as theoretical considerations, we find that a Higgs signal in the two photon channel with a rate equal to, or above, the SM prediction is viable over the full mass range $123 \mathrm{GeV} \lesssim M_{H} \lesssim 127 \mathrm{GeV}$, both in the MSSM and the NMSSM. We find that besides the interpretation of a possible signal at about $125 \mathrm{GeV}$ in terms of the lightest $\mathcal{C} \mathcal{P}$-even Higgs boson, both the MSSM and the NMSSM permit also a viable interpretation where an observed state at about $125 \mathrm{GeV}$ would correspond to the second-lightest $\mathcal{C P}$-even Higgs boson in the spectrum, which would be accompanied by another light Higgs with suppressed couplings to $W$ and $Z$ bosons. We find that a significant enhancement of the $\gamma \gamma$ rate, compatible with the signal strenghts observed by ATLAS and CMS, is possible in both the MSSM and the NMSSM, and we analyse in detail different mechanisms in the two models that can give rise to such an enhancement. We briefly discuss also our predictions in the two models for the production and subsequent decay into two photons of a $\mathcal{C P}$-odd Higgs boson.
\end{abstract}

*email: benbrik@ifca.unican.es

$\dagger$ email: melina@ifca.unican.es

$\ddagger$ email: Sven.Heinemeyer@cern.ch

$\S$ email: oscar.stal@desy.de

ฯemail: Georg.Weiglein@desy.de

" email: lisa.zeune@desy.de 


\section{Introduction}

Two of the most important goals of the experiments at the Large Hadron Collider (LHC) are to identify the origin of electroweak symmetry breaking, and to search for physics effects beyond the Standard Model (SM). The Higgs searches currently ongoing at the LHC (and previously carried out at the Tevatron) address both those goals. The spectacular discovery of a Higgs-like particle with a mass around $M_{H} \simeq 125 \mathrm{GeV}$, which has just been announced by ATLAS and CMS [1], marks a milestone of an effort that has been ongoing for almost half a century and opens a new era of particle physics. Both ATLAS and CMS reported a clear excess around $\sim 125 \mathrm{GeV}$ in the two photon channel as well as in the $Z Z^{(*)}$ channel, whereas the analyses in other channels have a lower mass resolution and at present are less mature. The combined sensitivity in each of the experiments reaches about $\sim 5 \sigma$. The observed rate in the $\gamma \gamma$ channel turns out to be considerably above the expectation for a SM Higgs both for ATLAS and CMS. While the statistical significance of this possible deviation from the SM prediction is not sufficient at present to draw a definite conclusion, if confirmed in the future it could be a first indication of a non-SM nature of the new state. The recent announcement follows the tantalising hints for an excess around $M_{H} \simeq 125 \mathrm{GeV}$ that had already been reported both by ATLAS [2] and CMS [3, 4]. The results of the SM Higgs searches at the Tevatron, based on the full dataset collected by CDF and D0 have also just been announced [5], showing a broad excess in the region around $M_{H} \sim 125 \mathrm{GeV}$ that reaches a significance of nearly $3 \sigma$ and would be compatible with a signal at about $M_{H} \sim 125 \mathrm{GeV}$.

The prime task now is clearly to study the properties of the discovered new particle and in particular to test whether the new particle is compatible with the Higgs boson of the SM or whether there are significant deviations from the SM predictions, which would point towards physics beyond the SM. The fact that the observed signal in the $H \rightarrow \gamma \gamma$ channel appears to be somewhat stronger than expected in the SM could be a first hint in this direction, although it is statistically not very significant up to now. This result nevertheless serves as a strong motivation for investigating possible alternatives to the SM where a possible signal in the $\gamma \gamma$ channel could be enhanced compared to the SM case.

One of the leading candidates for physics beyond the SM is supersymmetry (SUSY), which doubles the particle degrees of freedom by predicting two scalar partners for all SM fermions, as well as fermionic partners to all bosons. The most widely studied SUSY framework is the Minimal Supersymmetric Standard Model (MSSM) [6], which keeps the number of new fields and couplings to a minimum. The Higgs sector in particular contains two Higgs doublets, which in the $\mathcal{C P}$ conserving case leads to a physical spectrum consisting of two $\mathcal{C P}$-even, one $\mathcal{C P}$-odd and two charged Higgs bosons.

Going beyond the MSSM, this model has a simple extension in the Next-to-Minimal Supersymmetric Standard Model (NMSSM), see e.g. [7] for reviews. A particularly appealing motivation for considering the NMSSM is that it provides a solution for naturally associating an adequate scale to the $\mu$ parameter appearing in the MSSM superpotential [8, 9]. In the NMSSM, the introduction of a new singlet superfield that only couples to the Higgs sector gives rise to an effective $\mu$-term, generated in a similar way as the Yukawa mass terms of fermions through its vacuum expectation value. The new field must be a gauge singlet, since the parameter $\mu$ carries no $S U(3)_{\mathrm{C}} \times S U(2)_{\mathrm{I}} \times U(1)_{\mathrm{Y}}$ quantum numbers. This effective 
$\mu$-term is linked dynamically to the electroweak scale. The additional degrees of freedom from the singlet add to the NMSSM particle spectrum. In the case where $\mathcal{C P}$ is conserved, which we assume throughout the paper, the states in the Higgs sector can now be classified as three $\mathcal{C} \mathcal{P}$-even Higgs bosons, $h_{i}(i=1,2,3)$, two $\mathcal{C P}$-odd Higgs bosons, $a_{j}(j=1,2)$, and the charged Higgs boson pair $H^{ \pm}$. In addition, the SUSY partner of the singlet Higgs (called the singlino) extends the neutralino sector (to a total of five neutralinos).

The extended parameter space of the NMSSM as compared to the MSSM case gives rise to a rich and interesting phenomenology, in particular related to states in the Higgs sector that can be relatively light. As an example, Higgs-to-Higgs decays can be open (as in some of the benchmarks presented in [10]), with potential consequences for Higgs searches at the LHC. This non-trivial phenomenology of the NMSSM led in fact to the situation that it has not been possible to establish a general "no-loose" theorem for NMSSM Higgs discovery at the LHC [11] (similar conclusions were obtained for the case of the MSSM with complex parameters, see e.g. [12].) Scenarios with a light $\mathcal{C} \mathcal{P}$-even Higgs boson with SM-like couplings to gauge bosons that however decays in an unusual way into a pair of very light $\mathcal{C P}$-odd Higgs bosons $a_{1}\left(m_{a_{1}}<2 m_{b}\right)$, sometimes called "ideal Higgs scenarios" [13], have found particular attention. The case of a light $\mathcal{C P}$-even Higgs in the NMSSM with suppressed couplings to gauge bosons has been discussed in [14. A summary of phenomenological results for the NMSSM Higgs sector can be found in [15]. The presence of an additional neutralino can have implications for dark matter, which may in this case contain a sizeable singlet fraction (see [7] and references therein) or - like the new Higgs bosons - be very light [16].

Inspired by the spectacular discovery of a new particle in the LHC data that manifests itself in particular as a signal of at least (but possibly higher than) SM strength in the $\gamma \gamma$ channel, we investigate the corresponding predictions in both the MSSM and the NMSSM, and compare them to the SM case. In particular, we evaluate the predictions for the production of a MSSM or NMSSM Higgs boson via gluon fusion, the main production channel at the LHC, followed by the decay into two photons, the channel with the largest significance in the results that have just been reported. As a main focus, we analyse potential enhancements of the production cross section times branching ratio over the corresponding SM prediction and we confront those predictions with the experimental data. We discuss in detail how an enhanced $\gamma \gamma$ rate can be realised in the MSSM, and which additional mechanisms for an enhancement can occur in the NMSSM. We find that sizable enhancements of the $\gamma \gamma$ rate are possible in both models, accompanied by or without a suppression of the $W W^{(*)}$ decay mode. It is interesting to note that in both models the signal can be interpreted either as the lightest $\mathcal{C} \mathcal{P}$-even Higgs or as the second-lightest $\mathcal{C} \mathcal{P}$-even Higgs. The latter interpretation would imply that there would be an additional Higgs boson present with a mass below $125 \mathrm{GeV}$ and suppressed couplings to gauge bosons.

This paper is organised as follows: in Sect. 2 we give a short introduction to the NMSSM and specify our notation. In Sect. 3 we describe the more technical issues of the loop calculations involved: the framework for one-loop calculations in the NMSSM, the tools used for the numerical evaluation of observables, and we discuss constraints on the NMSSM parameter space. The main results are given in Section 4. We show the numerical results on loop-induced Higgs production and decays in the MSSM and NMSSM, which are compared to corresponding results in the SM (where relevant). Finally, the model predictions are confronted with the most recent results from the LHC Higgs searches, namely the discovery of 
a Higgs-like particle, and the implications are discussed. Section 5 contains our conclusions.

\section{The NMSSM}

\subsection{General considerations}

Like the MSSM, the NMSSM contains two Higgs doublet superfields $\hat{H}_{1}, \hat{H}_{2}$, but in addition there is also a singlet scalar superfield $\hat{S} 1$ If one only considers the new interaction between the three scalar fields which gives rise to the effective $\mu$-term a Peccei-Quinn (PQ) symmetry is introduced. This may be avoided by considering a term cubic in the new singlet superfield, with a coupling strength $\kappa$. This cubic term explicitly breaks the PQ symmetry, providing a mass to the scalar $\mathcal{C P}$-odd (would be) axion. In order to reduce the interactions permitted in a general superpotential we assume a discrete $Z_{3}$ symmetry. In this so-called $Z_{3}$-symmetric version of the NMSSM the superpotential is assumed to be scale invariant (preventing terms linear or quadratic in $\hat{S}$ ), thus it takes the general form

$$
W^{\mathrm{NMSSM}}=y_{D} \hat{Q} \cdot \hat{H}_{1} \hat{D}^{c}+y_{L} \hat{L} \cdot \hat{H}_{1} \hat{E}^{c}+y_{U} \hat{Q} \cdot \hat{H}_{2} \hat{U}^{c}+\lambda \hat{S} \hat{H}_{2} \cdot \hat{H}_{1}+\frac{1}{3} \kappa \hat{S}^{3},
$$

where contraction of $S U(2)$ indices is denoted by a dot product, i.e. $\hat{Q} \cdot \hat{H} \equiv \epsilon_{i j} \hat{Q}^{i} \hat{H}^{j}$ with $\epsilon_{i j}=-\epsilon_{j i}$ and $\epsilon_{12}=1$. Assuming supersymmetry breaking by an unspecified mechanism, there are new soft breaking terms compared to the MSSM that need to be considered,

$$
V_{\mathrm{soft}}^{\mathrm{NMSSM}}=V_{\mathrm{soft}}^{\mathrm{MSSM}}+m_{S}^{2}|S|^{2}+\lambda A_{\lambda} S H_{2} \cdot H_{1}+\frac{1}{3} \kappa A_{\kappa} S^{3}+\text { h.c. }
$$

Ignoring the possibility of $\mathcal{C P}$ violation, as we do throughout this work, all parameters can be assumed real.

\subsection{The Higgs sector}

The explicit form of the Higgs potential is obtained from the Higgs $F$-terms, $D$-terms and the soft SUSY-breaking terms as $V_{H}=V_{F}+V_{D}+V_{\text {soft }}$. The $F$-terms arise from the first derivative of the $Z_{3}$-invariant superpotential given in Eq. (1). As the singlet $S$ is assumed to only couple to the Higgs, there is no coupling to the gauge bosons, and the $D$-terms are the same as in the MSSM. The soft breaking terms relevant to the Higgs sector are given in Eq. (2). Combining everything, we get the scalar Higgs potential of the NMSSM a: 2

$$
\begin{aligned}
V_{H}= & m_{1}^{2}\left|H_{1}\right|^{2}+m_{2}^{2}\left|H_{2}\right|^{2}+m_{S}^{2}|S|^{2}+\left|\lambda \varepsilon_{i j}\left(H_{2 i} H_{1 j}\right)+\kappa S^{2}\right|^{2}+|\lambda|^{2}|S|^{2}\left(\left|H_{1}\right|^{2}+\left|H_{2}\right|^{2}\right) \\
& +\left(\lambda A_{\lambda} \varepsilon_{i j}\left(H_{2 i} H_{1 j}\right) S+\frac{1}{3} \kappa A_{\kappa} S^{3}+\text { c.c. }\right)+\frac{1}{8}\left(g_{1}^{2}+g_{2}^{2}\right)\left(\left|H_{1}\right|^{2}-\left|H_{2}\right|^{2}\right)^{2}+\frac{1}{2} g_{2}^{2}\left|H_{1 i}^{*} H_{2 i}\right|^{2} .
\end{aligned}
$$

The indices $\{i, j\}=\{1,2\}$ refer to the respective Higgs doublet component (summation over $i$ and $j$ is understood). The $\mathcal{C P}$-invariant Higgs potential $V_{H}$ contains the real soft breaking

\footnotetext{
${ }^{1}$ Quantities with a hat denote superfields; fields without hats are their corresponding scalar components.

${ }^{2}$ We use a notation that closely follows the one specified in 17 for the MSSM.
} 
parameters $m_{1}^{2}, m_{2}^{2}, m_{S}^{2}, A_{\lambda}, A_{\kappa}$, as well as the superpotential trilinear couplings $\lambda$ and $\kappa$, and the $U(1)_{\mathrm{Y}}$ and $S U(2)_{\mathrm{I}}$ coupling constants $g_{1}$ and $g_{2}$.

After spontaneous symmetry breaking the two Higgs doublets can be expanded similarly to the MSSM. In components they are given by

$$
H_{1}=\left(\begin{array}{c}
v_{1}+\frac{1}{\sqrt{2}}\left(\phi_{1}-\mathrm{i} \chi_{1}\right) \\
-\phi_{1}^{-}
\end{array}\right), \quad H_{2}=\left(\begin{array}{c}
\phi_{2}^{+} \\
v_{2}+\frac{1}{\sqrt{2}}\left(\phi_{2}+\mathrm{i} \chi_{2}\right)
\end{array}\right)
$$

and the ratio of the two vacuum expectation values defines

$$
\tan \beta \equiv \frac{v_{2}}{v_{1}}
$$

The complex scalar singlet can likewise be expanded as

$$
S=v_{s}+\frac{1}{\sqrt{2}}\left(\phi_{s}+\mathrm{i} \chi_{s}\right)
$$

According to Eq. (11) a non-zero vacuum expectation value $v_{s}$ for the singlet gives rise to an effective $\mu$ parameter (cf. the bilinear term $W_{2} \sim \mu \hat{H}_{2} \cdot \hat{H}_{1}$ in the MSSM superpotential)

$$
\mu_{\mathrm{eff}}=\lambda v_{s} .
$$

Since the scale for $\mu_{\text {eff }}$ is set by $v_{s}$, a value at the electroweak scale is natural. Minimising the Higgs potential, the mass parameters $m_{1}^{2}$ and $m_{2}^{2}$ appearing in Eq. (3) are replaced by the vacuum expectation values $v_{1}$ and $v_{2}$ (or, equivalently, $v=\sqrt{v_{1}^{2}+v_{2}^{2}} \simeq 174 \mathrm{GeV}$ and $\tan \beta)$. Similarly, the singlet soft mass $m_{S}^{2}$ can be expressed in terms of $v_{s}$.

The bilinear part of the Higgs potential is then given by

$V_{H}=\frac{1}{2}\left(\phi_{1}, \phi_{2}, \phi_{S}\right) \mathbf{M}_{\phi \phi \phi}\left(\begin{array}{c}\phi_{1} \\ \phi_{2} \\ \phi_{S}\end{array}\right)+\frac{1}{2}\left(\chi_{1}, \chi_{2}, \chi_{S}\right) \mathbf{M}_{\chi \chi \chi}\left(\begin{array}{c}\chi_{1} \\ \chi_{2} \\ \chi_{S}\end{array}\right)+\left(\phi_{1}^{-}, \phi_{2}^{-}\right) \mathbf{M}_{\phi^{ \pm} \phi^{ \pm}}\left(\begin{array}{c}\phi_{1}^{+} \\ \phi_{2}^{+}\end{array}\right)+\cdots$,

with the mass matrices $\mathbf{M}_{\phi \phi \phi}, \mathbf{M}_{\chi \chi \chi}$ and $\mathbf{M}_{\phi^{ \pm} \phi^{ \pm}}$for the three $\mathcal{C} \mathcal{P}$-even neutral scalars $\left(\phi_{1}, \phi_{2}, \phi_{s}\right)$, three $\mathcal{C P}$-odd neutral scalars $\left(\chi_{1}, \chi_{2}, \chi_{s}\right)$, and two charged pairs $\left(\phi_{1}^{ \pm}, \phi_{2}^{ \pm}\right)$, respectively. At tree-level, the elements of the mass matrix $\mathbf{M}_{\phi \phi \phi}$ for the $\mathcal{C P}$-even Higgs bosons is given in the basis $\left(\phi_{1}, \phi_{2}, \phi_{s}\right)$ by

$$
\begin{aligned}
& \left(\mathbf{M}_{\phi \phi \phi}\right)_{11}=M_{Z}^{2} \cos ^{2} \beta+B \mu_{\mathrm{eff}} \tan \beta \\
& \left(\mathbf{M}_{\phi \phi \phi}\right)_{22}=M_{Z}^{2} \sin ^{2} \beta+B \mu_{\mathrm{eff}} \cot \beta \\
& \left(\mathbf{M}_{\phi \phi \phi}\right)_{33}=\frac{\lambda^{2} v^{2} A_{\lambda}}{\mu_{\mathrm{eff}}} \cos \beta \sin \beta+K \mu_{\mathrm{eff}}\left(A_{\kappa}+4 K \mu_{\mathrm{eff}}\right), \\
& \left(\mathbf{M}_{\phi \phi \phi}\right)_{12}=\left(2 \lambda^{2} v^{2}-M_{Z}^{2}\right) \cos \beta \sin \beta-B \mu_{\mathrm{eff}}, \\
& \left(\mathbf{M}_{\phi \phi \phi}\right)_{13}=\lambda v\left[2 \mu_{\mathrm{eff}} \cos \beta-\left(B+K \mu_{\mathrm{eff}}\right) \sin \beta\right] \\
& \left(\mathbf{M}_{\phi \phi \phi}\right)_{23}=\lambda v\left[2 \mu_{\mathrm{eff}} \sin \beta-\left(B+K \mu_{\mathrm{eff}}\right) \cos \beta\right]
\end{aligned}
$$

where we have introduced $K \equiv \kappa / \lambda$ and $B \equiv A_{\lambda}+K \mu_{\mathrm{eff}}$, and $M_{Z}$ denotes the mass of the $Z$ boson. 
The $\mathcal{C P}$-odd mass matrix is simplified significantly by introducing an effective doublet mass

$$
\hat{m}_{A}^{2}=\frac{B \mu_{\mathrm{eff}}}{\sin \beta \cos \beta},
$$

which corresponds to the mass of the single $\mathcal{C P}$-odd Higgs boson in the MSSM limit. In the basis $\left(\chi_{1}, \chi_{2}, \chi_{s}\right)$ this gives

$$
\begin{aligned}
& \left(\mathbf{M}_{\chi \chi \chi}\right)_{11}=\hat{m}_{A}^{2} \sin ^{2} \beta \\
& \left(\mathbf{M}_{\chi \chi \chi}\right)_{22}=\hat{m}_{A}^{2} \cos ^{2} \beta \\
& \left(\mathbf{M}_{\chi \chi \chi}\right)_{33}=\frac{\lambda^{2} v^{2}}{\mu_{\mathrm{eff}}}\left(B+3 K \mu_{\mathrm{eff}}\right) \cos \beta \sin \beta-3 A_{\kappa} K \mu_{\mathrm{eff}}, \\
& \left(\mathbf{M}_{\chi \chi \chi}\right)_{12}=\hat{m}_{A}^{2} \sin \beta \cos \beta, \\
& \left(\mathbf{M}_{\chi \chi \chi}\right)_{13}=\lambda v\left(B-3 K \mu_{\mathrm{eff}}\right) \sin \beta, \\
& \left(\mathbf{M}_{\chi \chi \chi}\right)_{23}=\lambda v\left(B-3 K \mu_{\mathrm{eff}}\right) \cos \beta
\end{aligned}
$$

for the elements of the $\mathcal{C} \mathcal{P}$-odd mass matrix.

The mass eigenstates in lowest order follow from unitary transformations of the original fields,

$$
\left(\begin{array}{l}
h_{1} \\
h_{2} \\
h_{3}
\end{array}\right)=U^{H} \cdot\left(\begin{array}{l}
\phi_{1} \\
\phi_{2} \\
\phi_{S}
\end{array}\right), \quad\left(\begin{array}{l}
a_{1} \\
a_{2} \\
G
\end{array}\right)=U^{A} \cdot\left(\begin{array}{c}
\chi_{1} \\
\chi_{2} \\
\chi_{S}
\end{array}\right), \quad\left(\begin{array}{l}
H^{ \pm} \\
G^{ \pm}
\end{array}\right)=U^{C} \cdot\left(\begin{array}{l}
\phi_{1}^{ \pm} \\
\phi_{2}^{ \pm}
\end{array}\right) .
$$

The matrices $U^{H}, U^{A}$ and $U^{C}$ transform the neutral $\mathcal{C P}$-even, $\mathcal{C} \mathcal{P}$-odd and charged Higgs fields, respectively, such that the resulting mass matrices

$$
\mathbf{M}_{h h h}^{\text {diag }}=U^{H} \mathbf{M}_{\phi \phi \phi} U^{H^{\dagger}}, \mathbf{M}_{a a G}^{\text {diag }}=U^{A} \mathbf{M}_{\chi \chi \chi} U^{A^{\dagger}} \text { and } \mathbf{M}_{H^{ \pm} G^{ \pm}}^{\text {diag }}=U^{C} \mathbf{M}_{\phi^{ \pm} \phi^{ \pm}} U^{C^{\dagger}}
$$

are diagonal in the basis of the transformed fields. The new fields correspond to the three neutral $\mathcal{C P}$-even Higgs bosons $h_{1}, h_{2}$ and $h_{3}$ with $m_{h_{1}} \leq m_{h_{2}} \leq m_{h_{3}}$, the two $\mathcal{C P}$-odd Higgs bosons $a_{1}$ and $a_{2}$ with $m_{a_{1}} \leq m_{a_{2}}$, the charged pair $H^{ \pm}$and the Goldstone bosons $G$ and $G^{ \pm}$. The tree-level mass of the physical charged Higgs boson is related to the $\mathcal{C P}$-odd doublet mass through (with $M_{W}$ denoting the mass of the $W$ boson)

$$
M_{H^{ \pm}}^{2}=\hat{m}_{A}^{2}+M_{W}^{2}-\lambda^{2} v^{2},
$$

which shows that $M_{H^{ \pm}}$may be lower than its corresponding MSSM value.

\subsection{The neutralino and chargino sectors}

In addition to the extended scalar sector, the supersymmetric partner of the singlet Higgs field - the singlino $\tilde{S}$-leads to the presence of fifth neutralino in the NMSSM spectrum. As 
a result, the neutralino mass matrix in the basis $\left(\tilde{B}, \tilde{W}^{0}, \tilde{H}_{1}^{0}, \tilde{H}_{2}^{0}, \tilde{S}\right)$ is given at tree-level by

$$
\mathbf{M}_{\tilde{\chi}^{0}}=\left(\begin{array}{ccccc}
M_{1} & 0 & -g_{1} \frac{v_{1}}{\sqrt{2}} & g_{1} \frac{v_{2}}{\sqrt{2}} & 0 \\
0 & M_{2} & g_{2} \frac{v_{1}}{\sqrt{2}} & -g_{2} \frac{v_{2}}{\sqrt{2}} & 0 \\
-g_{1} \frac{v_{1}}{\sqrt{2}} & g_{2} \frac{v_{1}}{\sqrt{2}} & 0 & -\mu_{\mathrm{eff}} & -\lambda v_{2} \\
g_{1} \frac{v_{2}}{\sqrt{2}} & -g_{2} \frac{v_{2}}{\sqrt{2}} & -\mu_{\mathrm{eff}} & 0 & -\lambda v_{1} \\
0 & 0 & -\lambda v_{2} & -\lambda v_{1} & 2 K \mu_{\mathrm{eff}}
\end{array}\right) .
$$

It can be diagonalised by a single unitary (complex) matrix $N$ such that

$$
\operatorname{diag}\left(m_{\tilde{\chi}_{1}^{0}}, m_{\tilde{\chi}_{2}^{0}}, \ldots\right)=N^{*} \mathbf{M}_{\tilde{\chi}^{0}} N^{\dagger}
$$

is real, positive definite, and with the mass eigenvalues ordered as $m_{\tilde{\chi}_{i}^{0}} \leq m_{\tilde{\chi}_{j}^{0}}$ for $i<j$.

Since no new charged degrees of freedom are introduced, the chargino sector of the NMSSM is identical to that in the MSSM.

\subsection{Squarks}

The squark sector of the NMSSM is unchanged with respect to the MSSM. Assuming minimal flavour violation and $\mathcal{C P}$ conservation, the mass matrix of the two squarks of the same flavour, $\tilde{q}_{L}$ and $\tilde{q}_{R}$, is given by

$$
\mathbf{M}_{\tilde{q}}=\left(\begin{array}{cc}
M_{\tilde{q}_{L}}^{2}+m_{q}^{2}+M_{Z}^{2} \cos 2 \beta\left(I_{3}^{q}-Q_{q} s_{\mathrm{w}}^{2}\right) & m_{q} X_{q} \\
m_{q} X_{q} & M_{\tilde{q}_{R}}^{2}+m_{q}^{2}+M_{Z}^{2} \cos 2 \beta Q_{q} s_{\mathrm{w}}^{2}
\end{array}\right),
$$

with

$$
X_{q}=A_{q}-\mu_{\mathrm{eff}}\{\cot \beta, \tan \beta\}
$$

where $\{\cot \beta, \tan \beta\}$ applies for up- and down-type squarks, respectively. In these equations, $M_{\tilde{q}_{L}}, M_{\tilde{q}_{R}}$ are (real) soft SUSY-breaking masses (as a consequence of $S U(2)$ symmetry, $M_{\tilde{q}_{L}}$ is equal for the members of an $S U(2)$ doublet; in the numerical analysis below we assume a universal value $M_{\mathrm{SUSY}}=M_{\tilde{q}_{L}}=M_{\tilde{q}_{R}}$ ), while $A_{q}$ is the soft SUSY-breaking trilinear coupling and $\mu_{\text {eff }}$ the effective $\mu$ parameter as defined above. $I_{3}^{q}$ and $Q_{q}$ denote the weak isospin and the electric charge of the quark, respectively, and $s_{\mathrm{w}}=\sqrt{1-c_{\mathrm{w}}^{2}}$ with $c_{\mathrm{w}}=M_{W} / M_{Z}$. The squark mass eigenstates are obtained by the unitary transformation

$$
\left(\begin{array}{c}
\tilde{q}_{1} \\
\tilde{q}_{2}
\end{array}\right)=\mathbf{U}_{\tilde{q}}\left(\begin{array}{c}
\tilde{q}_{L} \\
\tilde{q}_{R}
\end{array}\right)
$$

with

$$
U_{\tilde{q}}=\left(\begin{array}{cc}
\cos \theta_{\tilde{q}} & \sin \theta_{\tilde{q}} \\
-\sin \theta_{\tilde{q}} & \cos \theta_{\tilde{q}}
\end{array}\right), \quad U_{\tilde{q}} U_{\tilde{q}}^{\dagger}=\mathbb{1}
$$


and the mass eigenvalues are given by

$$
\begin{aligned}
m_{\tilde{q}_{1,2}}^{2}=m_{q}^{2}+\frac{1}{2} & {\left[M_{\tilde{q}_{L}}^{2}+M_{\tilde{q}_{R}}^{2}+I_{3}^{q} M_{Z}^{2} \cos 2 \beta\right.} \\
& \left.\mp \sqrt{\left[M_{\tilde{q}_{L}}^{2}-M_{\tilde{q}_{R}}^{2}+M_{Z}^{2} \cos 2 \beta\left(I_{3}^{q}-2 Q_{q} s_{\mathrm{w}}^{2}\right)\right]^{2}+4 m_{q}^{2} X_{q}^{2}}\right] .
\end{aligned}
$$

\section{Higher-order corrections in the (N)MSSM}

\subsection{General considerations}

The investigations in the present paper are part of a bigger activity that we have undertaken in order to provide precise theoretical predictions for relevant observables in the NMSSM that can be used for comparing the NMSSM phenomenology with the SM, the MSSM and other scenarios of physics beyond the SM and for confronting the predictions with the available data. In order to investigate to what extent the Higgs search results can discriminate between different scenarios of physics at the Terascale, precise theoretical predictions both within the SM and possible alternatives of it are needed. In particular, if small deviations from the SM predictions are probed it is crucial to treat the considered model of new physics at the same level of precision to enable an accurate analysis and comparison. In the MSSM Higgs sector higher-order contributions are known to give numerically large effects (see, e.g., [18, 19]), and the same also holds for the NMSSM (see, e.g., [20]). For many observables it is therefore necessary to include corrections beyond leading order in the perturbative expansion to obtain reliable results. It is planned that the results presented in this paper together with other results in the NMSSM Higgs sector 21] will be implemented into a new version of the Fortran code FeynHiggs [17,22 24, which so far is restricted to predictions for Higgs physics in the SM and the MSSM. A few public codes already exist for numerical NMSSM calculations. The by far most widely used is NMSSMTools [25], which consists of subpackages to calculate the NMSSM spectrum, constraints on the parameter space, as well as Higgs decays and decay modes of sparticles [26]. Recently also an extension applicable to the NMSSM of the program SPheno [27] became available, which makes use of model implementations generated with SARAH [28].

Since the NMSSM extends the MSSM in the Higgs and the neutralino sectors, differences to the MSSM are best probed in these two sectors. The processes playing the main role in the reported discovery at the LHC, production via gluon fusion and decay into two photons, are in fact processes that are particularly sensitive to possible deviations between the SM, the MSSM and the NMSSM. The one-loop predictions for those processes correspond to the leading-order contributions, which are IR- and also UV-finite without renormalisation (for a recent discussion of the renormalisation of the NMSSM Higgs sector, see [29]), so that the set-up mentioned above can immediately be applied for the investigation of the processes that are more important for NMSSM Higgs phenomenology at the LHC. After the initial announcements of an excess in the LHC Higgs searches by ATLAS [2] and CMS [3] there was already a considerable activity in the literature concerning possible interpretations of the results in various versions of the MSSM [30 32], and also in the NMSSM [33 35]. We 
comment on specific differences and similarities between those results and ours below.

The calculation of loop diagrams, often involving a large number of fields, is a tedious and error-prone task if done by hand. This is true in particular for theories beyond the SM where the number of fields is significantly increased. For one-loop calculations, as will be the focus in the following, computer methods with a high degree of automation have been devised to simplify the work. However, most of the available tools so far have focused on calculations either in the SM or the MSSM. In order to facilitate loop calculations in the NMSSM, it is useful to employ the well-established public tools FeynArts [36], FormCalc [37, 38] and LoopTools [37, 39], with which one-loop calculations can be carried out with a high degree of automation. The program FeynArts [36] can be used to generate and draw the Feynman diagrams to a given order for the process under study, based on the information about the particle content and interactions that is supplied in a so-called model file. From the Feynman rules a mathematical expression for the corresponding amplitudes is generated. For one-loop amplitudes, the analytic simplifications, trace evaluation, tensor decomposition, etc. can then be carried out by FormCalc [37, 38, which combines the speed of FORM [40] with the more user-friendly interface of Mathematica. If the necessary information about the particles involved in the considered process (mass eigenvalues, mixing angles, etc.) is provided, the numerical evaluation of the FormCalc output can be carried out with LoopTools [37, 39]. In the standard distribution, FeynArts contains model files for the SM (including several variants), a general two-Higgs-doublet model, and an implementation of the MSSM.

With the goal of treating the NMSSM at the same level of accuracy as the MSSM, we are currently developing a framework that enables the computation of one-loop processes in the NMSSM in a highly automated way. This consists on the one hand of the input on the particle content, the interaction vertices, etc. that is needed for use with the packages FeynArts and LoopTools, and on the other hand of an appropriate renormalisation prescription for the NMSSM. In the present paper we will focus on loop-induced processes, for which no renormalisation is needed. A detailed discussion of our renormalisation prescription for the NMSSM will be presented elsewhere.

\subsection{Implementation}

As a first step we have compiled a new FeynArts model file for the NMSSM. Since it is foreseen that the same framework will be applied for several calculations beyond those presented in this paper, we describe our NMSSM implementation in some detail here. The basis for the model file itself — defining the particle content and interactions of the NMSSM (as described in Sect. 2) in a general $R_{\xi}$ gauge - is generated with the help of the program SARAH [28]. This program can be used to generate FeynArts model files, as well as output for many other programs, for any supersymmetric theory starting from its superpotential. For consistency checks, we also use an independent NMSSM model file generated with FeynRules [41. Starting from the output of SARAH we have introduced the standard nomenclature of FormCalc to activate its internal MSSM simplifications, applied unitarity relations to mixing matrices and couplings, and implemented some further improvements. These modifications, besides greatly improving the speed at which FormCalc performs one-loop calculations of NMSSM amplitudes, are essential for instance for verifying the cancellation of UV divergences at the algebraic level. 
To enable the numerical evaluation of observables, such as decay widths and cross sections, the analytic amplitudes of FormCalc can be exported to Fortran code. These must be supplemented with a so-called driver program to compute the necessary quantities (masses, mixings, etc.) from the fundamental parameters of the theory. The driver codes also provide standard facilities for numerical integration and the evaluation of master one-loop integrals through LoopTools. We have developed such a driver program for the NMSSM, which in its present state allows for Higgs and sparticle masses to be calculated either at tree-level (following Sect. 2), or using NMSSMTools (here we used version 2.3.5) [25] linked through a custom interface.

The NMSSM driver also offers the possibility to impose restrictions on the NMSSM parameter space resulting from the evaluation of various experimental or theoretical constraints. For instance, the constraints implemented in NMSSMTools can be accessed, and direct constraints on the extended Higgs sector are available through an interface to HiggsBounds [42. More details on the different constraints and how they are evaluated is given in Sect. 3.4 below.

\subsection{Verification}

We have performed several tests on the model file to verify the NMSSM implementation, in particular for the Higgs sector which is most relevant for the present work. The analytical expressions for the Feynman rules for the interaction vertices of the NMSSM obtained from SARAH have been compared to the independent FeynRules output. They have also been compared (analytically) in the MSSM limit to the corresponding vertices in the default MSSM implementation distributed with FeynArts.

A number of tree-level processes have been analysed numerically — including the decays of Higgs bosons and neutralinos - to test the mixing properties of the singlet state in the NMSSM. Comparing these to the results of NMSSMTools and NMSDECAY [26], we find overall good agreement with those previously obtained results after correcting for differences due to QCD corrections and the running of gauge couplings.

A further, extensive, and non-trivial test of the working NMSSM implementation is provided by the results for the processes that are induced at the one-loop level in the NMSSM. We have evaluated $\mathcal{O}(50) 1 \rightarrow 2$ processes and $\mathcal{O}(100) 2 \rightarrow 2$ processes of this type and checked them successfully for their UV- and IR-finiteness. This includes the Higgs production and decay modes that are phenomenologically most relevant at the LHC, which are described and analysed numerically in detail below. As an example we show in Fig. 1 generic Feynman diagrams contributing to $g g \rightarrow h_{i}$ (upper row), where $h_{i}$ denotes any neutral $\mathcal{C} \mathcal{P}$-even Higgs boson in the (N)MSSM, as well as to $h_{i} \rightarrow \gamma \gamma$ (lower row). These types of diagrams have been evaluated and used for our numerical analysis described in Sect. 4.

\subsection{Constraints on the parameter space}

Before moving on to our numerical analysis, we briefly discuss the various phenomenological constraints which exist on the parameter space of the NMSSM. As already mentioned, our computational framework allows for all these constraints to be evaluated and applied 

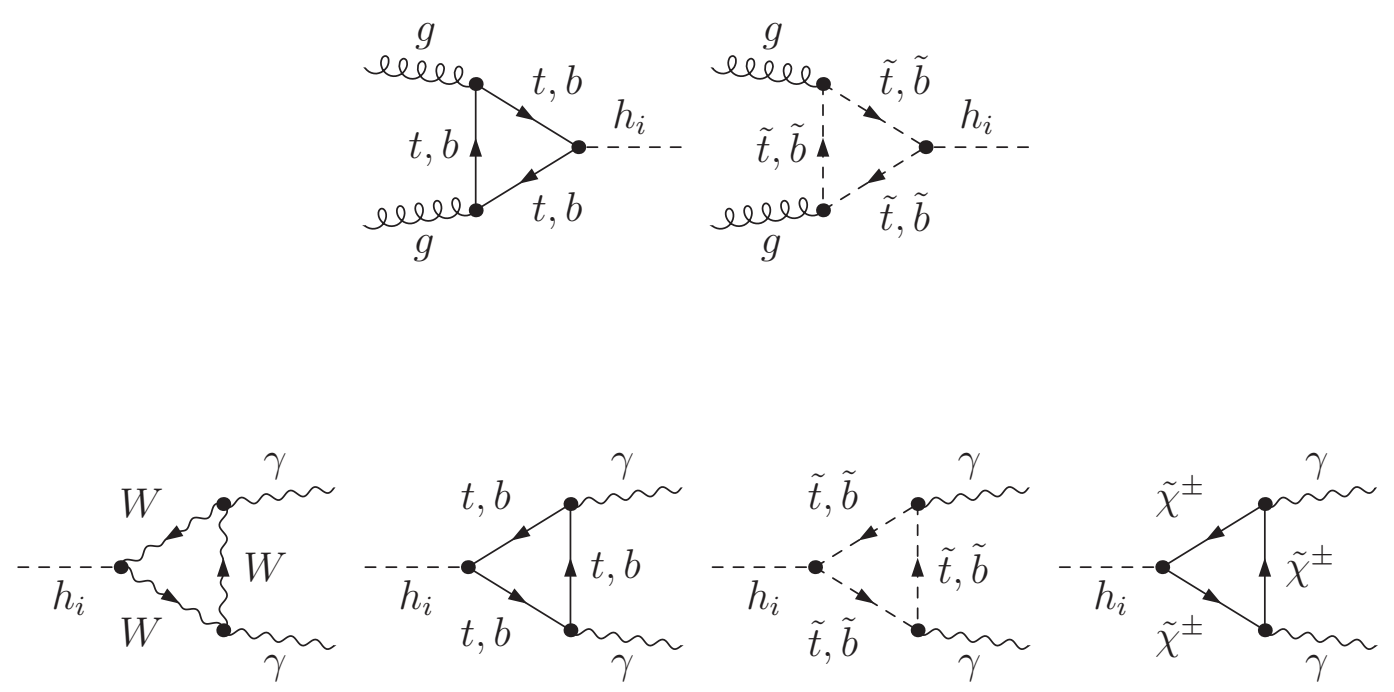

Figure 1: Generic diagrams contributing to $g g \rightarrow h_{i}$ (upper row) and to $h_{i} \rightarrow \gamma \gamma$ (lower row), where $h_{i}$ denotes any neutral $\mathcal{C} \mathcal{P}$-even Higgs boson in the (N)MSSM.

in connection with the calculation of arbitrary NMSSM observables. In this way reliable predictions can be obtained which are in agreement with the present experimental results.

\section{Theoretical constraints}

Constraints originate from the requirement of a viable physical minimum of the Higgs potential. The physical minimum, with non-vanishing vacuum expectation values (vevs) for the two Higgs doublets $H_{1}$ and $H_{2}$, should be lower than the local minimum with vanishing vevs for the two Higgs doublets. Furthermore the physical vacuum should have a non-zero singlet vev to be able to generate the $\mu_{\text {eff }}$ parameter. In the NMSSM, the correct pattern of a symmetry breaking absolute minimum is ensured approximately by the condition $A_{\kappa}^{2} \geq 9 m_{S}^{2}$ [43]. For each point considered in the NMSSM parameter space, we verified numerically (using NMSSMTools) that the Higgs potential is bounded from below and stable.

Another requirement is that there be no Landau pole for any of the running couplings $\lambda, \kappa, y_{t}$ and $y_{b}$ below $M_{\mathrm{GUT}} \approx 2 \times 10^{16} \mathrm{GeV}$. The renormalisation group equations for the NMSSM are known to two-loop order [44]. The constraint of perturbativity up to a very high scale restricts the range of $\lambda$ and $\kappa$. Values of these parameters in the perturbative regime at the GUT scale lead to comparably small values at the weak scale, which may be combined to give the approximate upper bound [9]

$$
\lambda^{2}+\kappa^{2} \leq 0.5
$$

In the parameter scan below we choose the ranges for $\lambda$ and $\kappa$ to respect this limit. 


\section{Bounds from direct Higgs searches}

The limits from Higgs searches at LEP, the Tevatron, and the 2011 LHC data put bounds on the Higgs masses and couplings. The LHC bounds have now been updated [1] and allow a SM-like Higgs boson only in a small window around $\sim 125 \mathrm{GeV}$. As strict bounds on the parameter space we will consider the data from LEP and the Tevatron as well as the LHC data presented in 2011 (referred to as $\mathrm{LHC}_{2011}$ ) and comment on the most recent limits as presented in [1] separately.

In the NMSSM, or any other theory with a Higgs sector different from that in the SM, the lower limit on the Higgs boson mass of $M_{H}>114.4 \mathrm{GeV}$ [45] from the LEP Higgs searches does not apply generically. The same is true for the limits from the searches for a SM-like Higgs at the $\mathrm{LHC}_{2011}$, which essentially rule out the mass range $127 \mathrm{GeV} \lesssim M_{H} \lesssim 600 \mathrm{GeV}$ for a SM-like Higgs. As has been demonstrated in various benchmark scenarios in the MSSM [46] and the NMSSM [47], allowed parameter regions for Higgs bosons of those extended Higgs sectors exist both below the LEP limit and above $127 \mathrm{GeV}$. In order to test whether a given point in the (N)MSSM parameter space is allowed or ruled out by the Higgs searches at LEP, the Tevatron, and the $\mathrm{LHC}_{2011}$ one therefore needs to confront the predictions of the model with the available cross section limits in the various search channels at each collider. For this purpose we make use of the code HiggsBounds [42] (version 3.6.1beta). In order to obtain the correct statistical interpretation in terms of a 95\% CL exclusion limit, HiggsBounds uses the input provided for the model under consideration (in the case of our analysis effective couplings and partial widths of the Higgs bosons of the MSSM and the NMSSM) to determine for each considered parameter point the channel that has the highest expected sensitivity for an exclusion. Only for this particular channel the theory prediction is then compared to the observed experimental limit, which determines in a statistically consistent manner whether the parameter point is allowed or excluded at the 95\% CL. The version of HiggsBounds used for our analysis includes in particular limits from CMS on an MSSM Higgs decay into a tau pair [48, which have a strong impact on the allowed region in the $\left(M_{A}, \tan \beta\right)$ plane 3 Results from $h \rightarrow \gamma \gamma$ [50,51] and $h \rightarrow W W$ channels [52,53] (among others) are also implemented 4 Due to their importance to our analysis, we will also display these limits separately below.

\section{Bounds from direct searches for SUSY particles}

Constraints also exist from direct searches for supersymmetric particles at LEP, the Tevatron, and the LHC. The least model-dependent limits are the ones from LEP. In particular the limit on the lightest chargino mass, $m_{\chi_{1}^{ \pm}}>94 \mathrm{GeV}$ [54, applies to both the MSSM and the NMSSM and restricts the parameter $\mu\left(\mu_{\text {eff }}\right)$ of the (N)MSSM to values above about $100 \mathrm{GeV}$.

\footnotetext{
${ }^{3}$ Using the latest limits from the CMS update [49] in this channel would lead to only minor changes in the results of our analysis.

${ }^{4}$ Strictly speaking, these results can only be directly interpreted in models with SM-like Higgs production. Even if this does not hold exactly, we have verified for the scenarios considered below that the ratio between the contributions from gluon fusion to vector boson fusion do not deviate from the SM case to the degree that vector boson fusion becomes the dominant production mode. Since we are mainly interested in investigating scenarios with an enhanced rate, we take a conservative approach and apply these bounds for maximal exclusion.
} 
For the squarks of the first two generations the LHC mass limits are most stringent [55], but have a certain model dependence. The ATLAS and CMS collaborations set limits above $1 \mathrm{TeV}$ in particular "simplified models" for the masses of first and second generation squarks and for the gluino; slightly weaker bounds are derived in the constrained MSSM. For the third generation squarks, which are most important for the radiative corrections in the Higgs sector, the bounds are much weaker and more model dependent. This means in particular that the presence of large stop mixing (as favoured by a relatively high value of the Higgs mass of about $125 \mathrm{GeV}$, see for instance Ref. [30]) is not excluded at present.

\section{The anomalous magnetic moment of the muon}

A significant deviation exists between the experimentally measured value of the anomalous magnetic moment of the muon, $a_{\mu}^{\exp }$ and the theoretical prediction in the SM. Numerically this deviation amounts to $\Delta a_{\mu}=a_{\mu}^{\exp }-a_{\mu}^{\mathrm{SM}}=(30.2 \pm 8.8) \times 10^{-10}$ [56, 57], which corresponds to more than a $3 \sigma$ effect. Employing new physics contributions to account for this deviation leads to bounds on the model parameters [58,59]. The dominant contributions to $a_{\mu}$ in the NMSSM are known including leading corrections up to the two-loop order [59]. For the numerical evaluation we use NMSSMTools. As the $2 \sigma$ allowed range for the NMSSM-specific contributions $\Delta a_{\mu}^{\mathrm{NMSSM}}=a_{\mu}^{\mathrm{NMSSM}}-a_{\mu}^{\mathrm{SM}}$ we use $1.21 \times 10^{-9}<\Delta a_{\mu}^{\mathrm{NMSSM}}<4.82 \times 10^{-9}$, which includes a theory uncertainty on the SUSY evaluation corresponding to $2.0 \times 10^{-10}$ added in quadrature to the uncertainty quoted above. We note that, similarly to the $\mu$ parameter in the MSSM, a positive value for $\mu_{\text {eff }}$ is strongly favoured when $a_{\mu}$ is included as a constraint.

\section{Flavour physics}

A recent analysis and summary of flavour physics constraints on the NMSSM parameter space has been presented in [47]. In the present setup we use NMSSMTools (version 2.3.5) to evaluate the NMSSM theory predictions. The corresponding experimental limits are listed in Table 1. Parameter-dependent theory uncertainties are added linearly to the intervals shown in the table before evaluating exclusion.

In theories with minimal flavour violation (MFV), which we are investigating here, the strongest constraints from flavour physics can usually be derived from $B$-physics observables such as $\operatorname{BR}\left(B \rightarrow X_{s} \gamma\right), \operatorname{BR}\left(B_{s} \rightarrow \mu^{+} \mu^{-}\right), \operatorname{BR}\left(B_{u} \rightarrow \tau^{+} \nu_{\tau}\right)$, or from the mass mixings $\Delta M_{s}$, $\Delta M_{d}$ [60,61]. In addition to the MSSM-type contributions from sparticles and non SM-like Higgs states (charged and neutral), the NMSSM may also be further constrained in the case where a very light $\mathcal{C} \mathcal{P}$-odd Higgs boson is present [62].

It has been argued that NMSSM specific contributions, i.e. contributions that go beyond the MSSM, to $\mathrm{BR}\left(B \rightarrow X_{s} \gamma\right)$ are negligible [61]. Besides the charged Higgs boson contribution, which typically yields large effects for a relatively small $M_{H^{ \pm}}$, we therefore include also the relevant loops of charginos, gluinos, as well as (non-singlet) neutralinos. Depending on the SUSY parameters, large cancellations between different contributions are possible, and any of the contributions mentioned above can become dominant. If a specific benchmark scenario is chosen for the SUSY parameters in the other sectors, it cannot be expected that such a cancellation takes place for all values of the Higgs sector parameters. However, a comparably small shift in the underlying SUSY scenario would typically be sufficient to 


\begin{tabular}{lccr}
\hline Observable & Exp. lower limit & Exp. upper limit & \\
\hline $\operatorname{BR}\left(B \rightarrow X_{s} \gamma\right)_{E_{\gamma}>1.6 \mathrm{GeV}}$ & $3.03 \times 10^{-4}$ & $4.07 \times 10^{-4}$ & {$[63$} \\
$\operatorname{BR}\left(B_{s} \rightarrow \mu^{+} \mu^{-}\right)$ & - & $1.1 \times 10^{-8}$ & 64 \\
$\operatorname{BR}\left(B^{ \pm} \rightarrow \tau \nu_{\tau}\right)$ & $0.79 \times 10^{-4}$ & $2.45 \times 10^{-4}$ & 65 \\
$\Delta M_{B_{s}}$ & $17.53 \mathrm{ps}^{-1}$ & $18.01 \mathrm{ps}^{-1}$ & 66 \\
$\Delta M_{B_{d}}$ & $0.499 \mathrm{ps}^{-1}$ & $0.515 \mathrm{ps}^{-1}$ & {$[63]$} \\
\hline
\end{tabular}

Table 1: Experimentally allowed ranges at the $2 \sigma$ level used for the flavour physics observables.

give rise to large compensations between the different contributions, which could bring the prediction into agreement with the experimental result.

Both the processes $\operatorname{BR}\left(B_{s} \rightarrow \mu^{+} \mu^{-}\right)$and $\operatorname{BR}\left(B_{u} \rightarrow \tau^{+} \nu_{\tau}\right)$ are also very sensitive to new physics effects, and can mainly exclude regions of parameter space with large $\tan \beta$. But while unacceptably large contributions to $\mathrm{BR}\left(B_{s} \rightarrow \mu^{+} \mu^{-}\right)$can be avoided in a similar fashion as for $\mathrm{BR}\left(B \rightarrow X_{s} \gamma\right)$ by a slight change of the other parameters of the SUSY scenario, such a compensation in general does not work for $\operatorname{BR}\left(B_{u} \rightarrow \tau^{+} \nu_{\tau}\right)$. This is due to the fact that this process involves charged Higgs exchange at tree-level. As a consequence, this process provides a similar exclusion power in the $\left(M_{H^{ \pm}}, \tan \beta\right)$ plane for the MSSM and the NMSSM.

\section{Numerical analysis}

In this section we analyse numerically the phenomenologically important loop-induced Higgs decays of the neutral $\mathcal{C} \mathcal{P}$-even Higgs bosons to two photons,

$$
h_{i} \rightarrow \gamma \gamma(i=1,2,3)
$$

For completeness, we also discuss the decays of the $\mathcal{C P}$-odd Higgs bosons,

$$
a_{j} \rightarrow \gamma \gamma(j=1,2)
$$

We investigate in particular, taking into account the existing constraints on the parameter space discussed above, to what extent the phenomenology of Higgs decays into two photons can differ in the MSSM and the NMSSM from the SM case. We choose a baseline MSSM benchmark scenario, fixing the soft SUSY-breaking parameters according to:

$$
\begin{aligned}
M_{\mathrm{SUSY}}=M_{\tilde{q}_{L}}=M_{\tilde{q}_{R}} & =1000 \mathrm{GeV} \\
M_{\tilde{l}_{L}}=M_{\tilde{l}_{R}} & =250 \mathrm{GeV}\left(\text { to comply with } \Delta a_{\mu}\right), \\
A_{t} & =A_{b}=A_{\tau}, \\
M_{1} & =\frac{5}{3} \tan ^{2} \theta_{\mathrm{w}} M_{2} \approx \frac{1}{2} M_{2}, \\
M_{2} & =400 \mathrm{GeV} \\
m_{\tilde{g}} & =1200 \mathrm{GeV} .
\end{aligned}
$$


While in the MSSM the tree-level Higgs sector can be specified by the two parameters $M_{H^{ \pm}}$ (or $M_{A}$ ) and $\tan \beta$, the NMSSM Higgs sector has larger freedom and requires additional input. We choose the following set of parameters to describe a point in the NMSSM parameter space:

$$
M_{H^{ \pm}}, \tan \beta, \lambda, K \equiv \kappa / \lambda, A_{\kappa} .
$$

The parameter $M_{H^{ \pm}}$here in principle plays the same role as in the MSSM. However, since we employ NMSSMTools to calculate the Higgs masses, the input $M_{H^{ \pm}}$is not defined in the on-shell renormalisation scheme, and must be understood as a tree-level input mass which can be directly translated to a value for $A_{\lambda}$ using Eqs. (10), (14). The calculated physical $M_{H^{ \pm}}$(including the higher order corrections) will therefore in general not be identical to the input value 5 The parameter $\mu_{\text {eff }}$, which we take as input, is in close correspondence to the $\mu$ parameter in the MSSM. Together with $\lambda$ it determines the value of the singlet vev through Eq. (7).

Below we are going to perform scans over the NMSSM parameter space. For the comparison between the NMSSM, the MSSM, and the SM we classify the resulting scenarios as follows:

(i) General NMSSM

This refers to any scenario in the NMSSM specified by the full set of parameters given by Eq. (26) and the MSSM parameters in Eq. (25) (where we also defined our default settings and values).

(ii) The MSSM limit

To recover the MSSM, we start from an arbitrary NMSSM scenario and take

$$
\lambda \rightarrow 0, \kappa \rightarrow 0, \quad K \equiv \kappa / \lambda=\text { constant. }
$$

All other parameters (including $\mu_{\text {eff }}$ ) are held fixed, which corresponds to $v_{s} \rightarrow \infty$. In this limit the Higgs doublet (and neutralino) couplings become MSSM-like, and the couplings of the singlets vanish. It should be noted, however, that the decoupled singlets do not necessarily correspond to the heaviest states $h_{3}, a_{2}$, and $\tilde{\chi}_{5}^{0}$ even in this limit. In the MSSM limit the parameters $M_{H^{ \pm}}$and $\tan \beta$ correspond exactly to their MSSM counterparts.

(iii) Decoupling (SM) limit

On top of the condition given by Eq. (27) for the MSSM limit, we define decoupling to the SM case by taking the additional limit

$$
M_{H^{ \pm}} \gg M_{Z} .
$$

This leads to heavy, nearly mass-degenerate, doublet Higgs states and leaves one light Higgs boson, $H$, with SM-like couplings to vector bosons and fermions. The singlet states are not affected by this limit; they remain decoupled with finite masses (which may or may not be lower than $M_{H}$ ).

\footnotetext{
${ }^{5}$ This feature would be avoided with an on-shell renormalisation of $M_{H^{ \pm}}$, see e.g. [17, 29].
} 


\begin{tabular}{ccc}
\hline Parameter & Minimum & Maximum \\
\hline$M_{\text {SUSY }}$ & 750 & 1500 \\
$M_{2} \simeq 2 M_{1}$ & 200 & 500 \\
$A_{t}=A_{b}=A_{\tau}$ & -2400 & 2400 \\
$\mu$ & 200 & 3000 \\
\hline$M_{A}$ & 100 & 600 \\
$\tan \beta$ & 1 & 60 \\
\hline
\end{tabular}

Table 2: Parameter ranges for the MSSM scan. All parameters with mass dimension are given in $\mathrm{GeV}$.

(iv) SM+singlet limit

When the doublet decoupling condition $M_{H^{ \pm}} \gg M_{Z}$ is fulfilled for points with finite non-zero $\lambda, \kappa$ (i.e., values that differ from the MSSM limit) we speak of the SM+singlet limit. This name is appropriate, since the low mass Higgs spectrum in this scenario consists of two $\mathcal{C P}$-even and one $\mathcal{C P}$-odd degree of freedom.

\subsection{Decays of $\mathcal{C P}$-even Higgs bosons in the MSSM}

Before we proceed to the NMSSM case, we study the two photon decays of the two $\mathcal{C} \mathcal{P}$-even Higgs bosons, $h$ and $H$, in the MSSM and compare to the SM. For the numerical evaluation in the MSSM we use the code FeynHiggs (version 2.8.6) [17,22 24, which is also used to evaluate SM quantities given in this section. To study interesting regions of the MSSM parameter space, where differences in the di-photon channel between the MSSM and the SM can occur, we perform a random scan over the parameter ranges given in Tab. 2. The remaining MSSM parameters are kept at their 'benchmark' values specified in Eq. (25).

It should be noted that we allow for comparably high values for $\mu$; this is relevant for the possible size of some of the effects that we will discuss in detail below. However, such large values of $\mu$, together with large values of $\tan \beta$, can lead to parameter combinations that show a non-stable behaviour in perturbation theory. In order to avoid parameter combinations that result in unacceptably large two-loop corrections in the evaluation of the Higgs boson self-energies and related quantities, we implement an upper limit on the corrections to the elements of the $\mathbf{Z}$ matrix (see Ref. [17]). Comparing the one- and two-loop values of the respective diagonal elements, we require the following condition for the light $\mathcal{C P}$-even Higgs, ||$Z_{11}^{2-\text { loop }}|-| Z_{11}^{1-\text { loop }}|| /\left|Z_{11}^{1-\text { loop }}\right|<0.25$, and analogously for the heavy $\mathcal{C} \mathcal{P}$-even Higgs with the replacement $Z_{11} \rightarrow Z_{22}$. We found that this upper bound is effective for avoiding parameter regions that are deemed unstable under higher-order corrections.

In Fig. 2 we show $\Gamma(h \rightarrow \gamma \gamma)$ in the top left and $\operatorname{BR}(h \rightarrow \gamma \gamma)$ in the bottom left plot as a function of $M_{h}$. The corresponding plots for $H \rightarrow \gamma \gamma$ are given in the right column. The colour coding is as follows: all points in the scan which are allowed by the theoretical constraints and the direct search limits for sparticles [54], as discussed above, are plotted in grey. Points which are also allowed by direct Higgs search limits (from HiggsBounds 3.6.1, i.e. including $\mathrm{LHC}_{2011}$ ) are shown in blue (on top of the grey points). Finally, points which fulfil additionally the constraint from $(g-2)_{\mu}$ and $\mathrm{BR}(b \rightarrow s \gamma)$ (both are here calculated with FeynHiggs) are plotted in black. The red (solid) curve in Fig. 2 shows the corresponding 

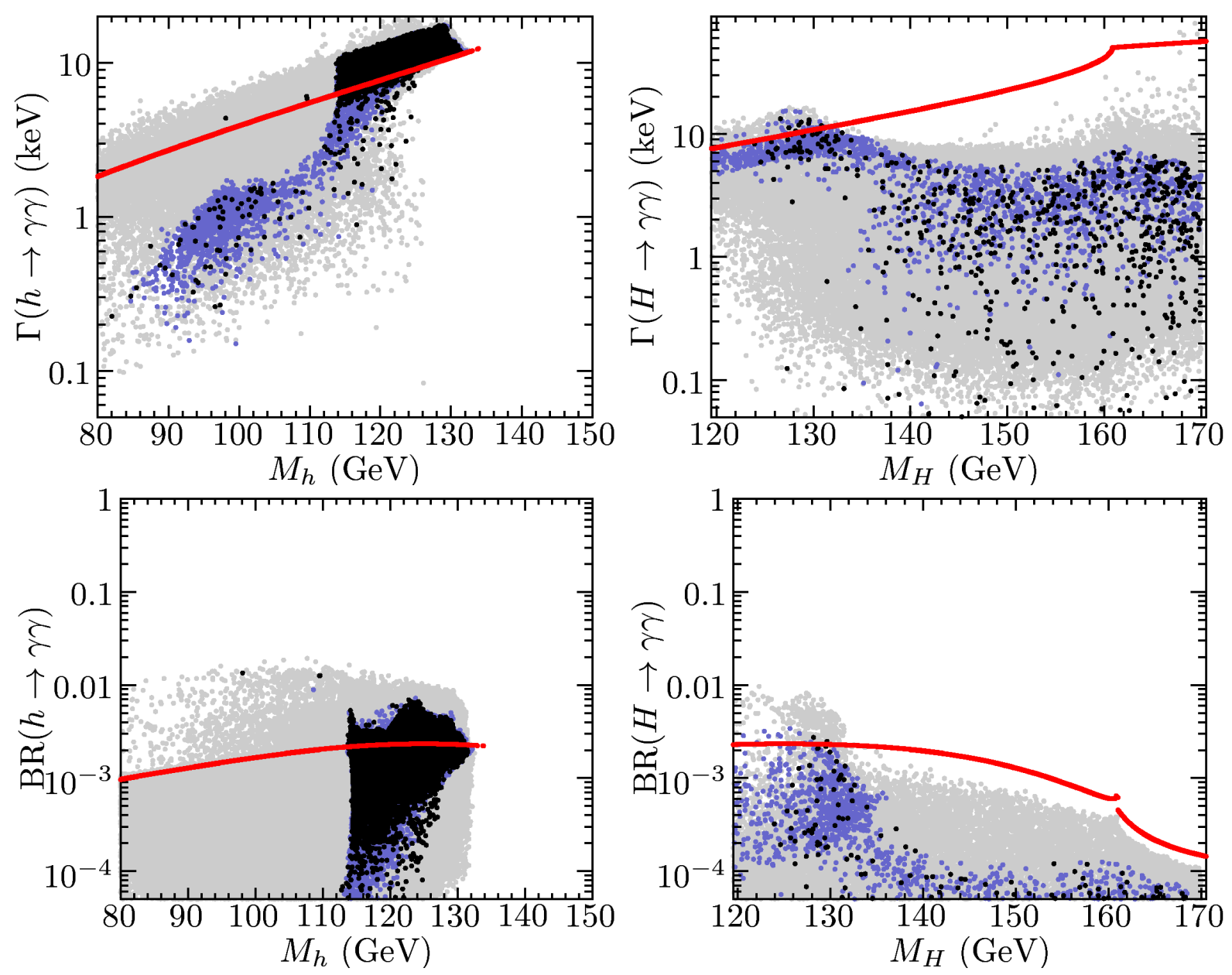

Figure 2: Results from the MSSM parameter scan for the partial widths $\Gamma(h, H \rightarrow \gamma \gamma)$ of $h$ (left) and $H$ (right), and the corresponding branching ratios. The full result of the scan (all points allowed by the theoretical constraints and the direct search limits for sparticles) is shown in grey. The blue points are compatible with the direct Higgs search limits (from HiggsBounds 3.6.1, i.e. including $\mathrm{LHC}_{2011}$ ), while the black points in addition give a result in agreement with $(g-2)_{\mu}$ and $\mathrm{BR}(b \rightarrow s \gamma)$. The solid (red) curve shows the respective quantities evaluated in the SM.

SM result with $M_{H_{\mathrm{SM}}}$ set equal to the corresponding MSSM Higgs mass. It should be noted that here (and in all the following plots) different densities of points appearing in different regions have no physical meaning, as the point density is related to the specific procedure chosen for the sampling of the SUSY parameter space.

We first focus on the light $\mathcal{C P}$-even Higgs boson, $h$, decaying into two photons. The extra particles in the MSSM yield additional loop contributions, which can both lower and raise $\Gamma(h \rightarrow \gamma \gamma)$ compared to the SM case. For $M_{h}<114.4 \mathrm{GeV} 6$ most of the scenarios where $\Gamma(h \rightarrow \gamma \gamma)>\Gamma\left(H_{\mathrm{SM}} \rightarrow \gamma \gamma\right)$ are ruled out by the direct Higgs search limits, but we also find allowed points in this region. For those $h$ couples with about SM strength to gauge

\footnotetext{
${ }^{6}$ We neglect here, and in the following, the theory uncertainty of the Higgs boson mass evaluation, which for the light Higgs boson should be roughly at the level of $2-3 \mathrm{GeV}$ [24].
} 
bosons, but is nevertheless not excluded due to a (much) suppressed coupling to $b$ quarks, which weakens the corresponding LEP limit. The fact that an enhanced rate for the decay of a Higgs boson to two photons is possible even below the LEP limit provides a motivation to extend the LHC Higgs searches to this region. In the following we focus on the mass region above the LEP limit. There we find scenarios in which $\Gamma(h \rightarrow \gamma \gamma)$ is enhanced by up to $\sim 70 \%$ with respect to the SM. On the other hand, as can be seen from the lower left plot in Fig. 2, the $\operatorname{BR}(h \rightarrow \gamma \gamma)$ can be enhanced by a factor $\sim 3$ over the SM in the same mass range (due to a suppression of the $b \bar{b}$ decay mode as discussed in more detail below). It is interesting to note that for the points that are allowed by all constraints the maximum enhancement of the branching ratio occurs around $M_{h} \sim 125 \mathrm{GeV}$. One can finally observe that no SM values are reached for $M_{h} \lesssim 114.4 \mathrm{GeV}$, reflecting the fact that a SM-like Higgs boson is ruled out by the LEP Higgs searches.

The corresponding results for the heavy $\mathcal{C} \mathcal{P}$-even MSSM Higgs boson are shown in the right column of Fig. 2. For $M_{H} \lesssim 130 \mathrm{GeV}$ we find viable points with a BR slightly larger than for a SM Higgs boson. For larger values of $M_{H}$ one can see the behaviour expected from the decoupling properties of the MSSM, i.e. $\Gamma(H \rightarrow \gamma \gamma)$ and $\operatorname{BR}(H \rightarrow \gamma \gamma)$ are both suppressed with respect to the SM, with the level of suppression increasing with $M_{H}$.

In order to investigate the phenomenology at the LHC, besides the branching ratio of course also the Higgs production cross section - which in general will also be modified in a model of physics beyond the SM - has to be taken into account. The combined enhancement or suppression over the SM for a process $p p \rightarrow h_{i} \rightarrow X$ can therefore be summarised in the ratio

$$
R_{X}^{h_{i}}=\frac{\sigma\left(p p \rightarrow h_{i}\right) \times \mathrm{BR}\left(h_{i} \rightarrow X\right)}{\sigma\left(p p \rightarrow H_{\mathrm{SM}}\right) \times \mathrm{BR}\left(H_{\mathrm{SM}} \rightarrow X\right)} .
$$

In the MSSM $h_{i}$ denotes either $h$ or $H$, while in the NMSSM (discussed in the next subsection) $h_{i}(i=1 \ldots 3)$ can be any of the three $\mathcal{C} \mathcal{P}$-even Higgs states. If the Higgs production cross section is dominated by a single mechanism, such as gluon fusion which is often the case at the LHC7, a common approximation is to use instead of $\sigma\left(p p \rightarrow h_{i}\right)$ the parton-level cross section $\hat{\sigma}\left(g g \rightarrow h_{i}\right)$. Neglecting the differences in kinematics, the decay width $\Gamma\left(h_{i} \rightarrow g g\right)$ has the same dependence as $\hat{\sigma}\left(g g \rightarrow h_{i}\right)$ on the couplings of the involved particles, and the dominant higher-order QCD corrections are expected to cancel out in the ratio 8 Making use of this approximation, Eq. (29) can be expressed as

$$
R_{X}^{h_{i}} \simeq \frac{\Gamma\left(h_{i} \rightarrow g g\right) \times \mathrm{BR}\left(h_{i} \rightarrow X\right)}{\Gamma\left(H_{\mathrm{SM}} \rightarrow g g\right) \times \mathrm{BR}\left(H_{\mathrm{SM}} \rightarrow X\right)}=\frac{\Gamma\left(h_{i} \rightarrow g g\right) \times \Gamma\left(h_{i} \rightarrow X\right) \times \Gamma_{\mathrm{tot}}\left(H_{\mathrm{SM}}\right)}{\Gamma\left(H_{\mathrm{SM}} \rightarrow g g\right) \times \Gamma\left(H_{\mathrm{SM}} \rightarrow X\right) \times \Gamma_{\mathrm{tot}}\left(h_{i}\right)} .
$$

This definition will be used to calculate $R_{\gamma \gamma}^{h_{i}}$ and $R_{W W}^{h_{i}}$ in the MSSM (and also in the NMSSM below).

7 We have checked that for the relevant regions of parameter space discussed below the gluon fusion production cross section always strongly dominates over the associated Higgs boson production from bottom quarks.

8 Non-negligible differences are mainly expected if the bottom loop contribution to $h_{i} \rightarrow g g$ dominates over the top loop contribution. In the case of the light $\mathcal{C} \mathcal{P}$-even Higgs boson can happen for very low $M_{A}$ and moderate to large $\tan \beta$ values, whereas in the case of the heavy $\mathcal{C} \mathcal{P}$-even Higgs boson this can 

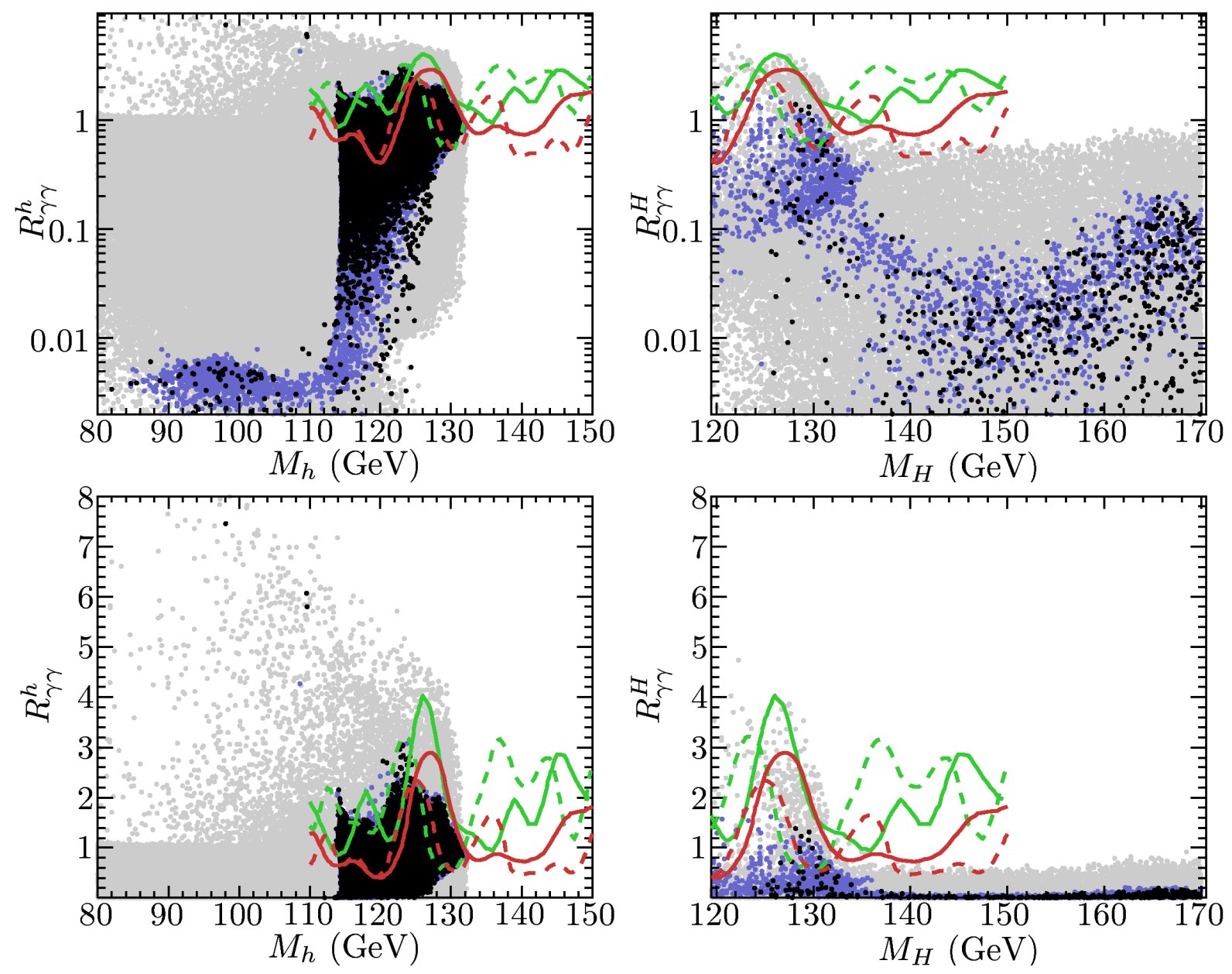

Figure 3: Results from the MSSM parameter scan on the ratios $R_{\gamma \gamma}^{h}$ for the light $\mathcal{C P}$-even Higgs boson $h$ (left column) and $R_{\gamma \gamma}^{H}$ for the heavy $\mathcal{C} \mathcal{P}$-even Higgs boson $H$ (right column). The plots are displayed both on a logarithmic scale (upper row) and on a linear scale (lower row). The colour coding for the scan points is the same as in Fig. 2. The green lines are the corresponding limits from data presented in 2011 data from ATLAS [50] (solid) and from CMS [51] (dashed). The red lines are the new limits from ATLAS (solid) and CMS (dashed) taken from [1].

The results for $R_{\gamma \gamma}^{h}$ and $R_{\gamma \gamma}^{H}$ are shown in Fig. 3, with the same colour coding as in Fig. 2. In order to make the results better visible we display them twice on a logarithmic scale (upper row) and on a linear scale (lower row). The green curves in Fig. 3 show exclusion limits in the di-photon channel at 95\% CL from data presented in 2011 data from ATLAS [50] (solid) and CMS [51] (dashed).9 The red lines are the new limits from ATLAS (solid) and CMS (dashed) taken from [1. The exclusion limits from ATLAS and CMS are displayed here

happen for larger $M_{A}$ and $\tan \beta \gtrsim 5$. Our results therefore exhibit an additional uncertainly in this part of the parameter space. Additional loop contributions from SUSY particles, while taken into account in our calculation, are usually subdominant and of lesser importance in this context.

${ }^{9}$ The 2011 exclusion limit from CMS in this channel was updated in 67. Including this limit in our analysis would not qualitatively change our results. 
explicitly for comparison, but only the $\mathrm{LHC}_{2011}$ data enters our analysis also as part of the constraints implemented in HiggsBounds. As explained above, HiggsBounds considers only the single channel with the highest expected sensitivity for determining 95\% CL (combined) exclusion. In the considered region the expected sensitivity of the CMS search [51] happens to be slightly higher than the one from ATLAS [50], so that only the CMS limit actually has an effect in our analysis. The plot shows also some allowed points with $R_{\gamma \gamma}^{h}$ above the CMS 2011 exclusion curve. For these points another channel has a higher expected sensitivity, so that the $\gamma \gamma$ channel has not been selected by HiggsBounds for determining the 95\% CL limit.

As one can see in the left column of Fig. 3, for $R_{\gamma \gamma}^{h}$ in principle a large enhancement, roughly up to a factor six, would be possible in the mass range $M_{h}=114 \ldots 130 \mathrm{GeV}$ (and an even stronger enhancement for lighter masses). Such large enhancements are now ruled out by the LHC searches in the $\gamma \gamma$ channel. For the points that are allowed by all the considered constraints we find that in the region above the LEP limit a suppression of $R_{\gamma \gamma}^{h}$ by more than an order of magnitude is possible. A maximal enhancement of about $\sim 3$ times the SM value, on the other hand, occurs for $M_{h} \approx 124 \mathrm{GeV}$. This is in interesting agreement with the recent data announcing a discovery of a new state compatible with a Higgs boson close to $\sim 125 \mathrm{GeV}$, that has been reported both by ATLAS and CMS. This observed excess is compatible with a SM Higgs signal, or even better with a signal of a somewhat enhanced strength in the two photon channel. Our results show that the MSSM could account for an enhanced Higgs signal as compared to the SM case around $M_{h}=125 \mathrm{GeV}$ with the maximal strength that is allowed by the present limits from ATLAS and CMS. The detailed origin of this enhancement will be discussed below. Fig. 3 also shows that the possible size of the enhancement decreases for larger $M_{h}$; for $M_{h}=130 \mathrm{GeV} R_{\gamma \gamma}^{h}$ is confined to values close to unity for the allowed points in the parameter space.

The right column of Fig. 3 shows the corresponding results for the heavy $\mathcal{C P}$-even Higgs. For $M_{H} \lesssim 130 \mathrm{GeV}$ the results for the heavy MSSM Higgs are qualitatively similar to the ones for the light $\mathcal{C P}$-even Higgs. In particular, also in this case a slight enhancement over the SM rate is possible for $M_{H} \approx 125 \mathrm{GeV}$ for the scan points that are in agreement with the collider constraints (as discussed above, the agreement with $(g-2)_{\mu}$ and the observables in the flavour sector could be improved by modifying some of the SUSY parameters that do not directly influence Higgs phenomenology). The size of the possible enhancement turns out to have some sensitivity to the condition that we have imposed on the elements of the $\mathbf{Z}$ matrix to ensure perturbatively reliable results, see above. Somewhat larger enhancements would be possible if the upper limit that we have imposed on the relative size of the twoloop corrections were relaxed. Our results for $R_{\gamma \gamma}^{H}$ demonstrate that the discovery of a new boson in the $\gamma \gamma$ channel at a mass of about $125 \mathrm{GeV}$ that was observed by ATLAS and CMS could also be interpreted within the MSSM as arising from the heavier $\mathcal{C} \mathcal{P}$-even Higgs boson, as discussed in [30]. Such a scenario would imply that besides a possible signal at about $125 \mathrm{GeV}$ there would be a lighter Higgs in the spectrum, having significantly suppressed couplings to gauge bosons. For $M_{H} \gtrsim 135 \mathrm{GeV}$ we always find $R_{\gamma \gamma}^{H}<1$, in accordance with the decoupling properties of the MSSM.

Before turning to a discussion of the specific mechanisms responsible for possible enhancements of the di-photon channel in the MSSM as compared to the SM case, we would like to make a brief comparison of our MSSM results to those existing in the literature. A previous 
study of the di-photon channel in the MSSM [68] found a smaller maximum enhancement, $R_{\gamma \gamma}^{h} \sim 1.5$, and only for a very specific Higgs mass region. We find a larger enhancement, primarily due to a larger scan range in $\mu$. Restricting our scan ranges accordingly, our results agree with those of [68]. Similarly, the analysis of [69] uses a more restricted range of $\mu<1 \mathrm{TeV}$, and we also find agreement with our results for $R_{\gamma \gamma}^{h}$ in this parameter region. In [70], it was claimed that a light Higgs boson with $M_{h} \approx 125 \mathrm{GeV}$ and $R_{\gamma \gamma}^{h} \gtrsim 1$ cannot be realised in the MSSM. Clearly, the results from our scan do not corroborate these conclusions since we find that such a Higgs boson can indeed be realised in the MSSM, possibly even with a rate enhanced compared to the SM.

The issue of a possible enhancement of $R_{\gamma \gamma}^{h}$ for a Higgs mass around $125 \mathrm{GeV}$ has also been discussed in [31], where in particular the contributions from light staus to $\mathrm{BR}(h \rightarrow \gamma \gamma)$ and the suppression of $h \rightarrow b \bar{b}$ due to Higgs mixing effects have been emphasised. As seen above, we find that $\Gamma(h \rightarrow \gamma \gamma)$ can exceed its SM value, which is found to be an effect of the stau loop contributions. The most sizeable enhancements observed in $R_{\gamma \gamma}^{h}$, however, mainly arise from a suppression of the total width, which in the SM is dominated by the partial decay width into $b \bar{b}$. Suppressing the $b \bar{b}$ channel can therefore yield a significant reduction of the total MSSM width. Such a suppression can happen in two different ways. The reduced $h b \bar{b}$ coupling in the MSSM is given at tree-level by

$$
\frac{g_{h b \bar{b}}}{g_{H_{\mathrm{SM}} b \bar{b}}}=-\frac{\sin \alpha}{\cos \beta},
$$

where $\alpha$ is the mixing angle in the $\mathcal{C} \mathcal{P}$-even Higgs sector. In the decoupling limit $\left(M_{A} \gg\right.$ $M_{Z}$ ) the $\mathrm{SM}$ is recovered, i.e. $(-\sin \alpha / \cos \beta) \rightarrow 1$. Higher-order contributions from Higgs propagator corrections can approximately be included via the introduction of an effective mixing angle, corresponding to the replacement $\alpha \rightarrow \alpha_{\text {eff }}$ [71] (in our numerical analysis we treat propagator-type corrections of the external Higgs bosons in a more complete way, which is based on wave function normalisation factors that form the $\mathbf{Z}$ matrix [17]). A suppression of the $h \rightarrow b \bar{b}$ channel thus occurs for small $\alpha_{\text {eff }}$. This feature motivated the definition of the "small $\alpha_{\text {eff }}$ " benchmark scenario, see [72]. In this scenario, the suppression of $\Gamma(h \rightarrow b \bar{b})$ occurs for large $\tan \beta$ and very small values of $M_{A}$, below $200 \mathrm{GeV}$.

Genuine corrections to the $h b \bar{b}$ vertex can lead to another type of suppression. Beyond leading order, loop-induced Yukawa couplings of $b$ quarks to the "wrong" Higgs doublet $H_{u}$ are induced. The modified $h b \bar{b}$ coupling can then be expressed as

$$
\frac{g_{h b \bar{b}}}{g_{H_{\mathrm{SM}} b \bar{b}}}=\frac{1}{1+\Delta_{b}}\left(-\frac{\sin \alpha_{\mathrm{eff}}}{\cos \beta}+\Delta_{b} \frac{\cos \alpha_{\mathrm{eff}}}{\sin \beta}\right) .
$$

Via the quantity $\Delta_{b}\left[73,74\right.$ terms of $\mathcal{O}\left(\left(\alpha_{s} \tan \beta\right)^{n}\right)$ and $\mathcal{O}\left(\left(\alpha_{t} \tan \beta\right)^{n}\right)$ can be resummed. The most relevant contributions are given by

$$
\Delta_{b}=\frac{2 \alpha_{s}\left(m_{t}\right)}{3 \pi} \tan \beta m_{\tilde{g}} \mu I\left(m_{\tilde{b}_{1}}^{2}, m_{\tilde{b}_{2}}^{2}, m_{\tilde{g}}^{2}\right)+\frac{\alpha_{t}\left(m_{t}\right)}{4 \pi} \tan \beta A_{t} \mu I\left(m_{\tilde{t}_{1}}^{2}, m_{\tilde{t}_{2}}^{2},|\mu|^{2}\right),
$$

with

$$
I(a, b, c)=-\frac{a b \ln (b / a)+a c \ln (a / c)+b c \ln (c / b)}{(a-c)(c-b)(b-a)}
$$




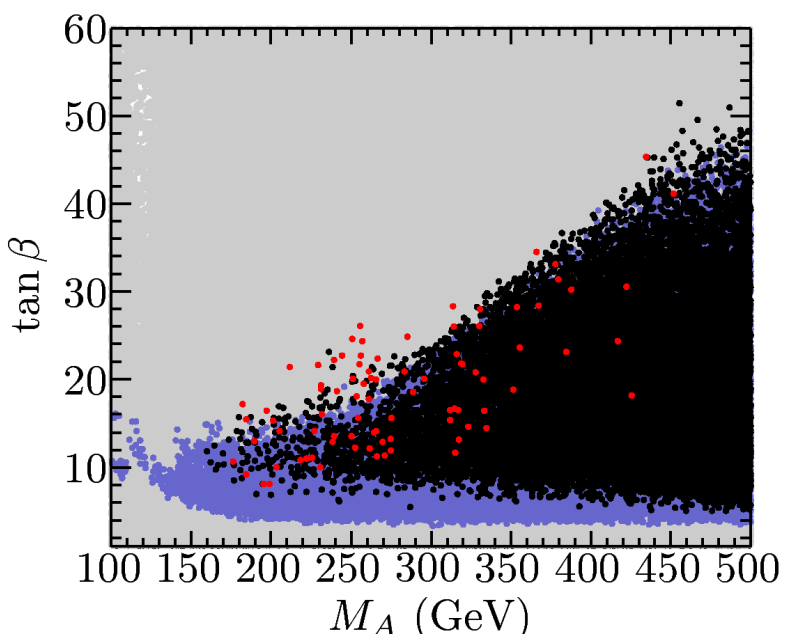

Figure 4: Results from the MSSM parameter scan on the ratio $R_{\gamma \gamma}^{h}$ in the $\left(M_{A}, \tan \beta\right)$ plane. The grey points are excluded by the limits from direct Higgs searches (from HiggsBounds 3.6.1, i.e. including $\mathrm{LHC}_{2011}$ ). MSSM Points with an enhancement of $R_{\gamma \gamma}^{h}$, corresponding to $R_{\gamma \gamma}^{h}>1$, are indicated in black, points with $R_{\gamma \gamma}^{h}>2$ are shown in red.

The dominant higher-order contribution to $\Delta_{b}$ are the QCD corrections, given in [75]. Those contributions are not included in our analysis, but their effect can be approximated by using a scale of $m_{t}$ for the evaluation of the one-loop expression, Eq. (33). While the loop-corrected $h b \bar{b}$ coupling, Eq. (32), approaches the tree-level coupling, Eq. (31), in the decoupling limit $\left(M_{A} \gg M_{Z}\right)$, a suppression of $g_{h b \bar{b}}$ is possible for not too large $M_{A}$ if $\Delta_{b}$ is numerically sizable and positive. For $\mu>1 \mathrm{TeV}$ we find enhancements of $R_{\gamma \gamma}^{h}$ of more than 1.5 for values of $M_{A}$ up to roughly $450 \mathrm{GeV}$ and with moderate to large values of $\tan \beta$. Points with $R_{\gamma \gamma}^{h}>2$ are possible if $\Delta_{b}$ is relatively large, $\Delta_{b} \simeq 0.5$. The corresponding effect on $R_{\gamma \gamma}^{h}$ can be seen in Fig. 4 for the $\left(M_{A}, \tan \beta\right)$ plane. The points with $R_{\gamma \gamma}^{h}>1$ are indicated in black, and the ones with $R_{\gamma \gamma}^{h}>2$ are shown in red. The regions with only grey points are excluded by the limits from the Higgs searches at LEP and the $\mathrm{LHC}_{2011}$, where the latter ones arise from the searches for MSSM Higgs bosons in the $\tau^{+} \tau^{-}$channel [4]. As can be seen from the figure, a slight enhancement of $R_{\gamma \gamma}^{h}>1$ can be accommodated over the whole allowed region displayed in Fig. 4, while scenarios with $R_{\gamma \gamma}^{h}>2$ tend to be closer to the boundary of the region allowed by the LHC Higgs limits.

We now turn to the alternative case where $h$ is light and has suppressed couplings to gauge bosons, whereas the heavier $\mathcal{C} \mathcal{P}$-even Higgs $H$ is a SM-like Higgs boson. One finds a similar enhancement for $R_{\gamma \gamma}^{H}$, which is due to the suppression of $g_{H b \bar{b}}$, if

$$
\frac{g_{H b \bar{b}}}{g_{H_{\mathrm{SM}} b \bar{b}}}=\frac{\cos \alpha}{\cos \beta}
$$

is small. Such an enhancement is restricted to the mass region $M_{H} \lesssim 130 \mathrm{GeV}$, since for higher mass values the coupling of the heavy $\mathcal{C} \mathcal{P}$-even Higgs to gauge bosons is suppressed, so that the partial width $\Gamma(H \rightarrow \gamma \gamma)$ is smaller than for the SM case, see Fig. 2. Accordingly, the scenarios with $R_{\gamma \gamma}^{H}>1$ are only realised in a relatively small parameter region close to the exclusion bounds from the Higgs searches, for $M_{A} \lesssim 150 \mathrm{GeV}$ and intermediate $\tan \beta$. 

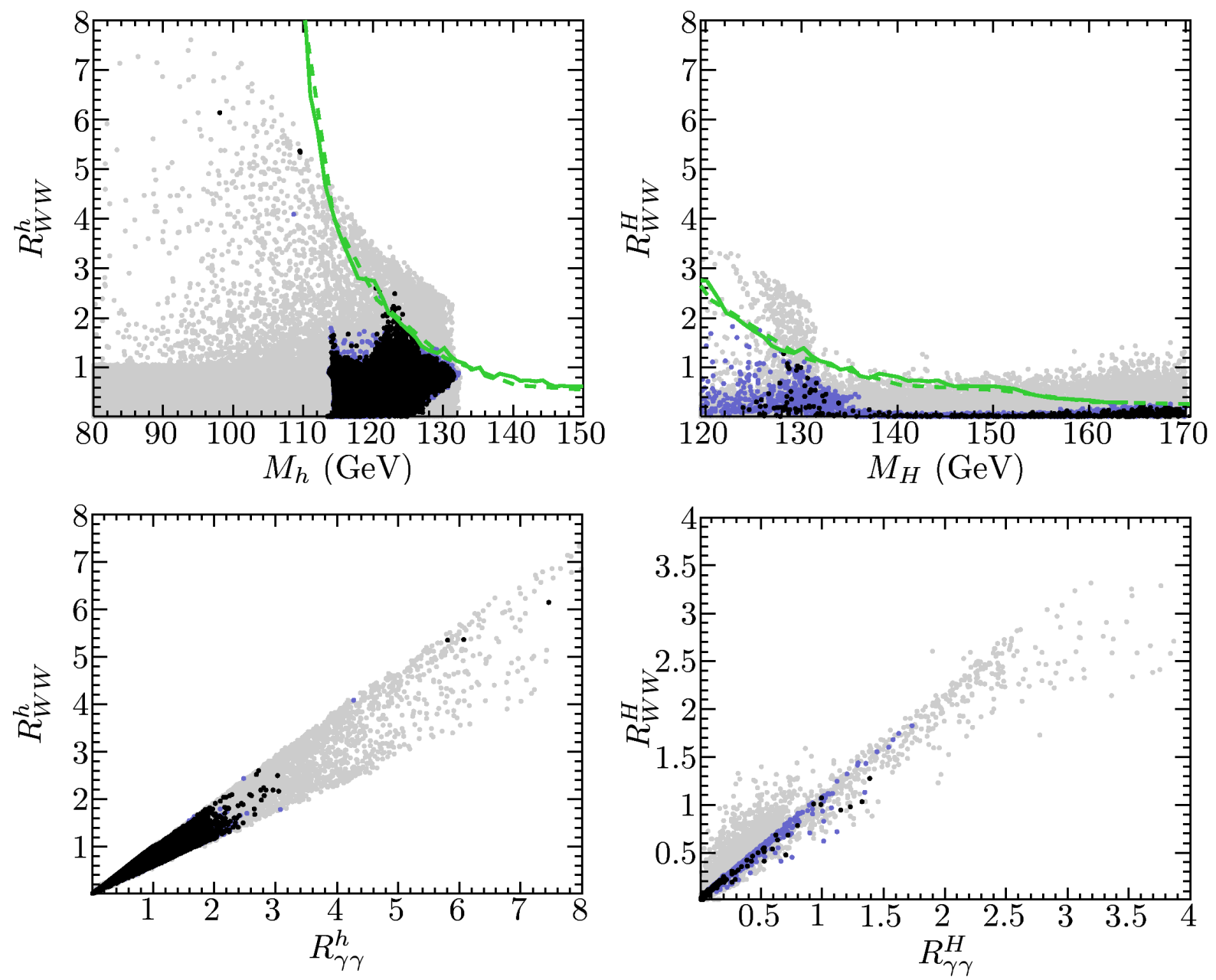

Figure 5: Results from the MSSM parameter scan on the ratios $R_{W W}^{h}$ for the light $\mathcal{C} \mathcal{P}$-even Higgs bosons $h$ (left column) and $R_{W W}^{H}$ for the heavy $\mathcal{C} \mathcal{P}$-even Higgs boson $H$ (right column). The plots in the upper row show $R_{W W}^{h}$ and $R_{W W}^{H}$ as a function of the respective Higgs mass. The $95 \%$ CL exclusion limits for the $W W^{(*)}$ channel using $\mathrm{LHC}_{2011}$ data from ATLAS [52] (solid line) and CMS 53. (dashed line) are also shown. The plots in the lower row show the correlation to $R_{\gamma \gamma}^{h}$ and $R_{\gamma \gamma}^{H}$. The colour coding is the same as in Fig. 2 ,

The scenario in which $R_{\gamma \gamma}^{H}$ is enhanced is complementary to the one giving an enhancement in $R_{\gamma \gamma}^{h}$ (as we checked explicitly). Consequently, a simultaneous enhancement in the di-photon channel for both $\mathcal{C P}$-even Higgs bosons is not possible.

A reduction of the total width, by the suppression of the $h, H \rightarrow b \bar{b}$ channel, can also affect the search for the Higgs boson in other channels. In Fig. 5 we investigate the decays $h, H \rightarrow W W^{(*)}$. The colour coding is as in Fig. 2, but here the green lines show the $95 \%$ CL exclusion limits for the data presented in 2011 for the $W W^{(*)}$ channel from ATLAS [52] (solid) and CMS [53] (dashed). Here we do not show new results from [1], since this channel was updated only by CMS, and no combination of 2011 and 2012 data is available. The 2012 exclusion curve of CMS is very similar to the one based on 2011 data. Similarly to $R_{\gamma \gamma}^{h}$, also $R_{W W}^{h}$ can be enhanced in the MSSM. The maximal enhancement of $R_{W W}^{h} \approx 2.5$ 

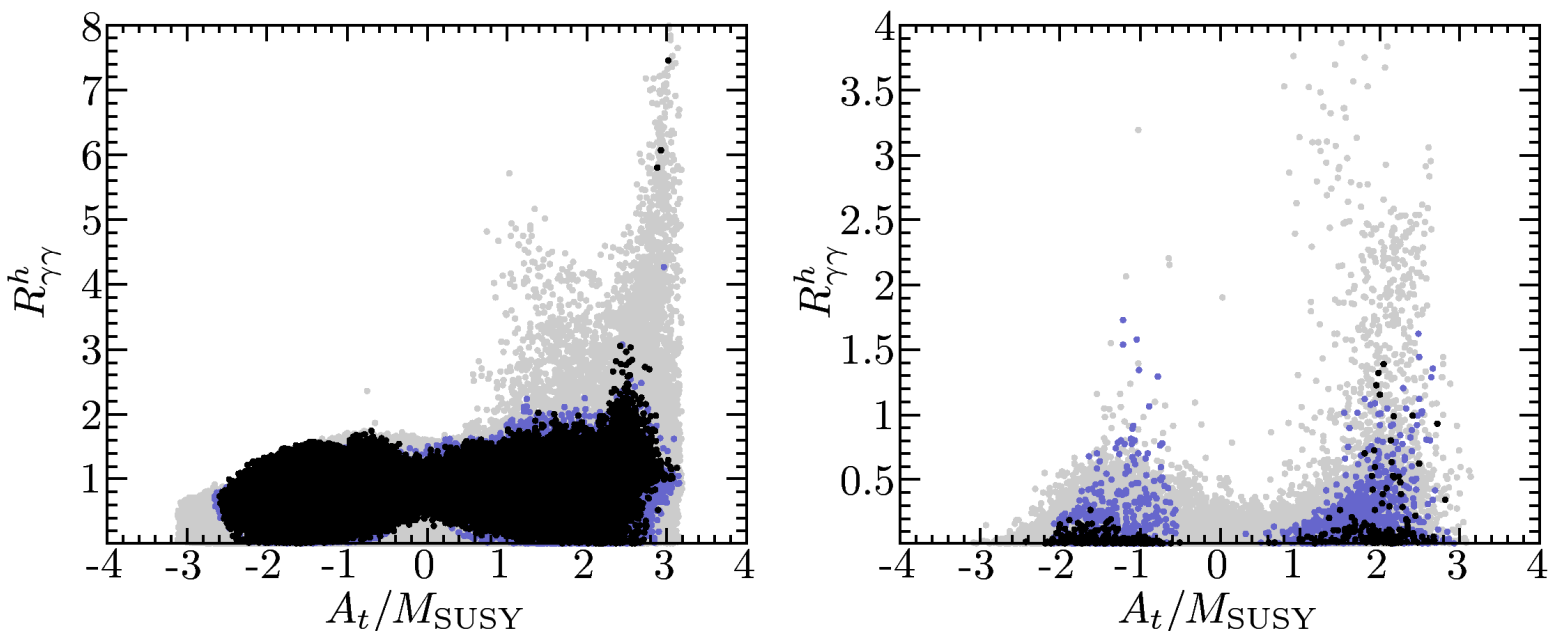

Figure 6: Results from the MSSM parameter scan on the ratios $R_{\gamma \gamma}^{h}$ (left) and $R_{\gamma \gamma}^{H}$ (right) as function of $A_{t} / M_{\mathrm{SUSY}}$. The colour code is as in Fig. 2,

that is compatible with the existing bounds is found for $M_{h} \approx 125 \mathrm{GeV}$. These points, above the green lines, are not excluded by the $h \rightarrow W W^{(*)}$ limits, because another channel has a higher expected sensitivity for those points (and consequently the $W W^{(*)}$ channel has not been selected by HiggsBounds for determining the 95\% CL limit - which would not change by the inclusion of the 2012 data shown in [1]). Since the main contribution to $h \rightarrow \gamma \gamma$ comes from diagrams containing $W$-boson loops, a strong correlation between these two channels is expected. This correlation is confirmed by plots in the lower row of Fig. 5. Contrary to $\Gamma(h \rightarrow \gamma \gamma), \Gamma\left(h \rightarrow W W^{(*)}\right)$ does not exceed its SM value, and therefore the maximal enhancement of $R_{W W}^{h}$ is always smaller than the enhancement of $R_{\gamma \gamma}^{h}$. This leaves some room to have e.g. a SM-like rate, or even a rate that is somewhat suppressed w.r.t. the SM, for $h \rightarrow W W^{(*)}$ (and $h \rightarrow Z Z^{(*)}$ ) with a simultaneous enhancement in $h \rightarrow \gamma \gamma$. Concerning the heavy $\mathcal{C P}$-even Higgs boson, the results are qualitatively similar, but with a smaller possible enhancement of the $\gamma \gamma$ or $W W^{(*)}$ rate, as we show in the right column of Fig. 5 .

Finally, in the left (right) plot of Fig. [6 we show the dependence of $R_{\gamma \gamma}^{h}\left(R_{\gamma \gamma}^{H}\right)$ as a function of $A_{t} / M_{\mathrm{SUSY}}$, with the colour coding as in Fig. 2. Here it should be kept in mind that values of $\left|A_{t}\right| \gtrsim \sqrt{6} M_{\text {SUSY }}$ could potentially lead to charge or colour breaking minima [76]. For the light $\mathcal{C P}$-even Higgs, as shown in the left plot, the largest values of $R_{\gamma \gamma}^{h}$ are indeed found around $A_{t} \sim \sqrt{6} M_{\text {SUSY }}$. However, even applying a stringent cut $\left|A_{t}\right|<\sqrt{6} M_{\text {SUSY }}$ would still leave allowed points with $R_{\gamma \gamma}^{h} \sim 2$. For the heavy $\mathcal{C P}$-even Higgs, we see that a stringent cut on $\left|A_{t}\right|$ would leave nearly all points with $R_{\gamma \gamma}^{H}>1$.

\section{$4.2 \mathcal{C P}$-even Higgs decays in the NMSSM}

We now turn to the NMSSM and analyse the di-photon decay in this model. For a light $\mathcal{C P}$-even Higgs boson with a mass below $114.4 \mathrm{GeV} 10$ it was pointed out in [77] that a strong

10 As before, we neglect here and in the following, the theory uncertainty of the Higgs boson mass evaluation, which for the light Higgs boson should be comparable to or slightly larger than the respective 
enhancement of the $\gamma \gamma$ rate - up to a factor of seven over the SM rate - is possible. In light of the recent results, the main focus of our analysis will naturally be on higher Higgs masses. For other approaches regarding an interpretation of a possible Higgs signal around $M_{h}=125 \mathrm{GeV}$ in terms of an NMSSM Higgs boson, see [33 35].

As before we consider the one-loop induced Higgs decay $h_{1,2} \rightarrow \gamma \gamma$, but now calculated using the framework described in Sect. 3. We perform a scan over the NMSSM parameter space and evaluate the partial widths and branching ratios for this mode. The parameter ranges used for the scan are given in Tab. 3. The remaining parameters are fixed to the benchmark scenario defined above, see Eq. (25). It should be noted that the ranges in Tab. 3 are not meant to cover the full NMSSM parameter space. The effects discussed above that can cause an enhancement of $R_{\gamma \gamma}^{h, H}$ in the MSSM can be realised also in the context of the NMSSM. In the present analysis we are interested in genuine NMSSM effects, which go beyond the MSSM phenomenology. Such genuine NMSSM effects arise in particular from the mixing of the Higgs doublet fields with the Higgs singlet. To be specific, we consider scenarios that are characterised by large values of $M_{H^{ \pm}}$, corresponding to the "SM+singlet" limit of the NMSSM discussed above. We furthermore restrict $\mu_{\text {eff }}$ and $\tan \beta$ to relatively small values, while our MSSM scan (cf. Tab. 2) had extended to rather large values of $\mu$ and $\tan \beta$ and had focussed on the region of relatively low values of $M_{A}$. The parameters are chosen such that the mechanisms for enhancing $R_{\gamma \gamma}^{h, H}$ realised in the MSSM do not play a role, putting the emphasis on the genuine NMSSM effects.

\begin{tabular}{cccc}
\hline Parameter & Minimum & Maximum & \\
\hline$A_{t}=A_{b}=A_{\tau}$ & -2400 & 2400 & $\mathrm{GeV}$ \\
$\mu_{\mathrm{eff}}$ & 150 & 250 & $\mathrm{GeV}$ \\
\hline$M_{H^{ \pm}}$ & 500 & 1000 & $\mathrm{GeV}$ \\
$\tan \beta$ & 2.6 & 6 & \\
\hline$\lambda$ & 0.5 & 0.7 & \\
$K$ & 0.3 & 0.5 & \\
$A_{\kappa}$ & -100 & -5 & $\mathrm{GeV}$ \\
\hline
\end{tabular}

Table 3: Parameter ranges used for the $\mathcal{C} \mathcal{P}$-even Higgs decay scan in the NMSSM.

The results for $h_{1,2} \rightarrow \gamma \gamma$ are shown in Fig. $7\left(h_{3}\right.$ is always heavy and plays no role in our analysis). The colour coding is the same as in Fig. 2, i.e. all displayed points satisfy the "theoretical" constraints of Sect. 3.4 (grey). Points which in addition fulfil the direct Higgs exclusion limits from colliders (from HiggsBounds 3.6.1, i.e. including $\mathrm{LHC}_{2011}$ ) are drawn in blue, and points which satisfy all the constraints, in particular also those from $\Delta a_{\mu}$ and flavour physics, are shown in black. The red curve shows the corresponding SM result, obtained by setting $M_{H_{\mathrm{SM}}}=m_{h_{1,2}}$, respectively.

We choose to study $m_{h_{1}}$ in a range from $80 \mathrm{GeV}$ up to its maximum around $135 \mathrm{GeV}$. Allowed points with $m_{h_{1}}<80 \mathrm{GeV}$ are also found in the scan, but the large singlet component of these very light Higgs bosons gives rise to a quite different phenomenology, which we do not investigate in detail here. For masses close to $140 \mathrm{GeV}$, the number of allowed points is seen to decrease, which illustrates that only quite specific choices of the input parameters give uncertainty of the MSSM, i.e. roughly at the level of $2-3 \mathrm{GeV}[24$. 

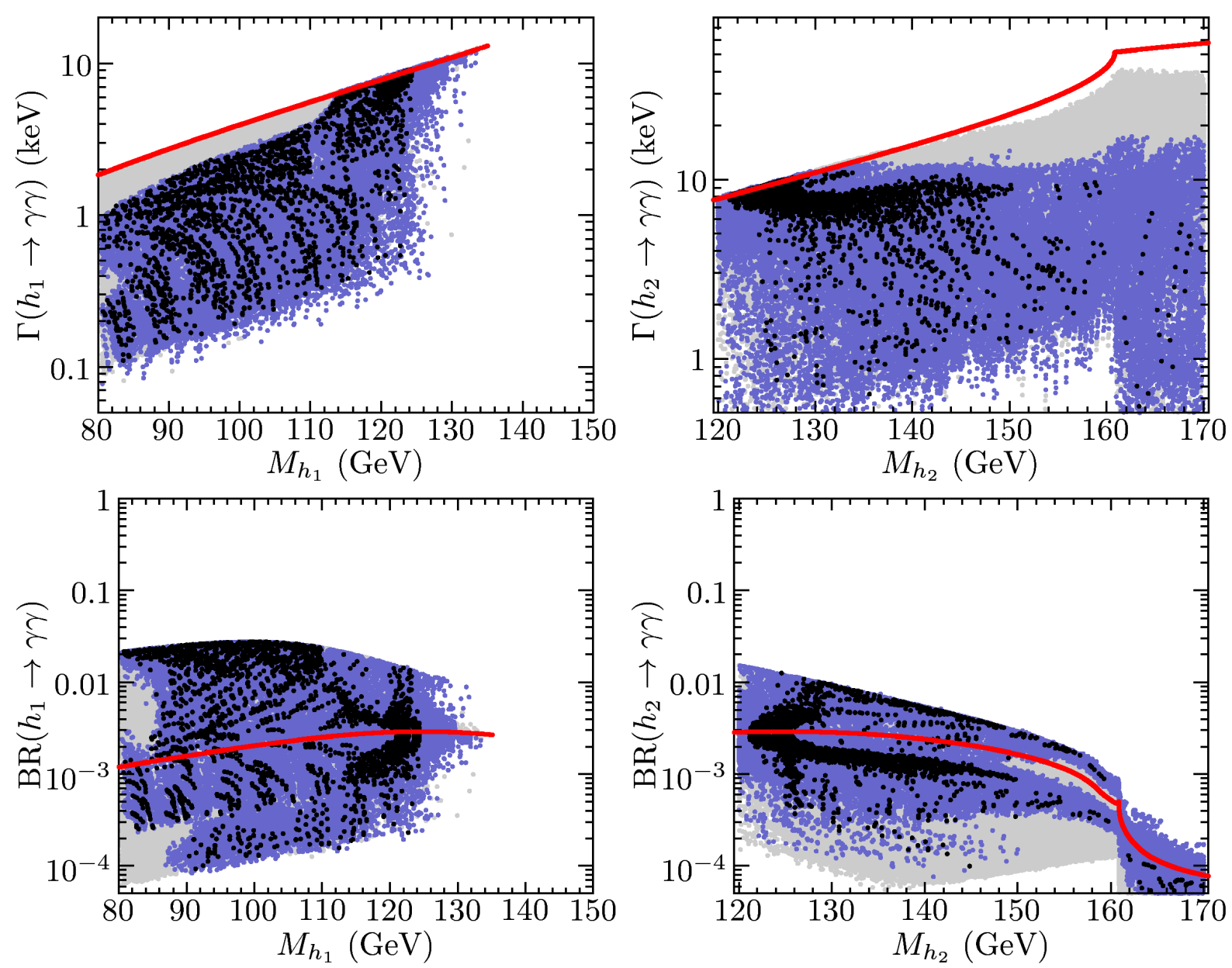

Figure 7: Results from the NMSSM parameter scan (see text) for the partial widths $\Gamma\left(h_{i} \rightarrow\right.$ $\gamma \gamma$ ) and the corresponding branching ratios of $h_{1}$ (left) and $h_{2}$ (right). All points in the figure fulfil the "theoretical" constraints defined in Sect. 3.4. In addition, the blue (dark) points satisfy direct Higgs search limits from colliders (from HiggsBounds 3.6.1, i.e. including $\mathrm{LHC}_{2011}$ ), while the black points are in agreement with all theoretical and experimental constraints. The solid (red) curve shows the respective quantities evaluated in the SM.

$m_{h_{1}}$ close to the maximum. This, as well as other features with local under- (over-) density of points in certain regions, can simply be viewed as sampling artefacts, i.e. the point density has no physical meaning. For $h_{2}$ we study the mass interval $120 \mathrm{GeV}<m_{h_{2}}<170 \mathrm{GeV}$, which means there is an overlap with the region considered for $m_{h_{1}}$. To go even higher in $m_{h_{2}}$ is not particularly interesting for our purposes, since when the two-body decay $h_{2} \rightarrow W W^{(*)}$ is open the loop-induced $h_{2} \rightarrow \gamma \gamma$ decay becomes suppressed (as is also clearly visible in the figure).

Fig. [7 shows that $\Gamma\left(h_{i} \rightarrow \gamma \gamma\right)$ is always smaller than (or at most equal to) its SM value for the points in our scan. This means in particular that our scan, for which we have fixed the slepton masses to large values (see Eq. (25)), does not contain points with light staus (the contribution of light staus was discussed in the MSSM context above). For $m_{h_{2}} \gtrsim 140 \mathrm{GeV}$, the partial width does not reach the full SM value, which shows that this mass region is 

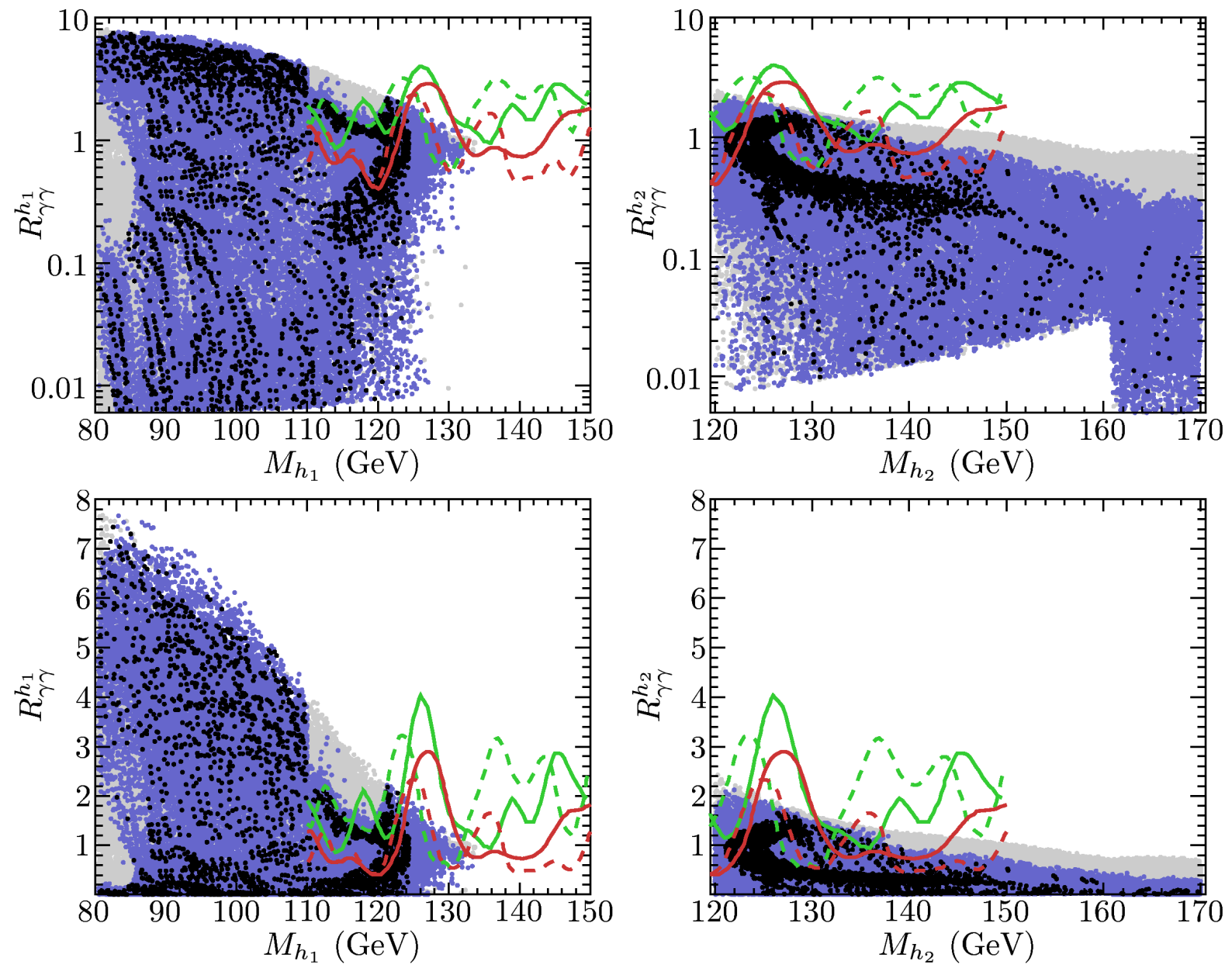

Figure 8: Results from the NMSSM parameter scan on the ratio $R_{\gamma \gamma}^{h_{i}}$ for the two lightest Higgs bosons $h_{1}$ (left column) and $h_{2}$ (right column). The plots are displayed both on a logarithmic scale (upper row) and on a linear scale (lower row). The colour coding for the scan points is the same as in Fig. 17. The green lines show exclusion limits on this channel at $95 \% \mathrm{CL}$ from $\mathrm{LHC}_{2011}$ data from ATLAS [50] (solid) and from CMS [51] (dashed). The red lines are the new limits from ATLAS (solid) and CMS (dashed) taken from [1].

not accessible for a fully SM-like $h_{2}$. Taking into account the collider constraints, we also see that, as in the MSSM, a SM-like Higgs boson with $m_{h_{1}}<114.4 \mathrm{GeV}$ is excluded as a consequence of the LEP limits. Despite the smaller NMSSM width for $\Gamma\left(h_{i} \rightarrow \gamma \gamma\right)$ compared to the SM, Fig. 7 shows that an enhancement of the branching ratio with up to an order of magnitude over the SM is possible. The results are similar for $h_{1}$ and $h_{2}$ in the overlapping mass region.

As in the case of the MSSM we now analyse $R_{\gamma \gamma}^{h_{i}}$. The total widths appearing in Eq. (30) are calculated in an approximate way according to

$$
\Gamma_{\text {tot }}\left(h_{i}\right)=\frac{1}{m_{h_{i}}} \operatorname{Im}\left[\Sigma_{h_{i}}\left(m_{h_{i}}^{2}\right)\right]+\Gamma\left(h_{i} \rightarrow W W^{(*)}\right)+\Gamma\left(h_{i} \rightarrow \gamma \gamma\right)+\Gamma\left(h_{i} \rightarrow g g\right),
$$

where $\Sigma_{h_{i} h_{i}}$ denotes the one loop self energy of $h_{i}$. The inclusion of the off-shell decays, as 

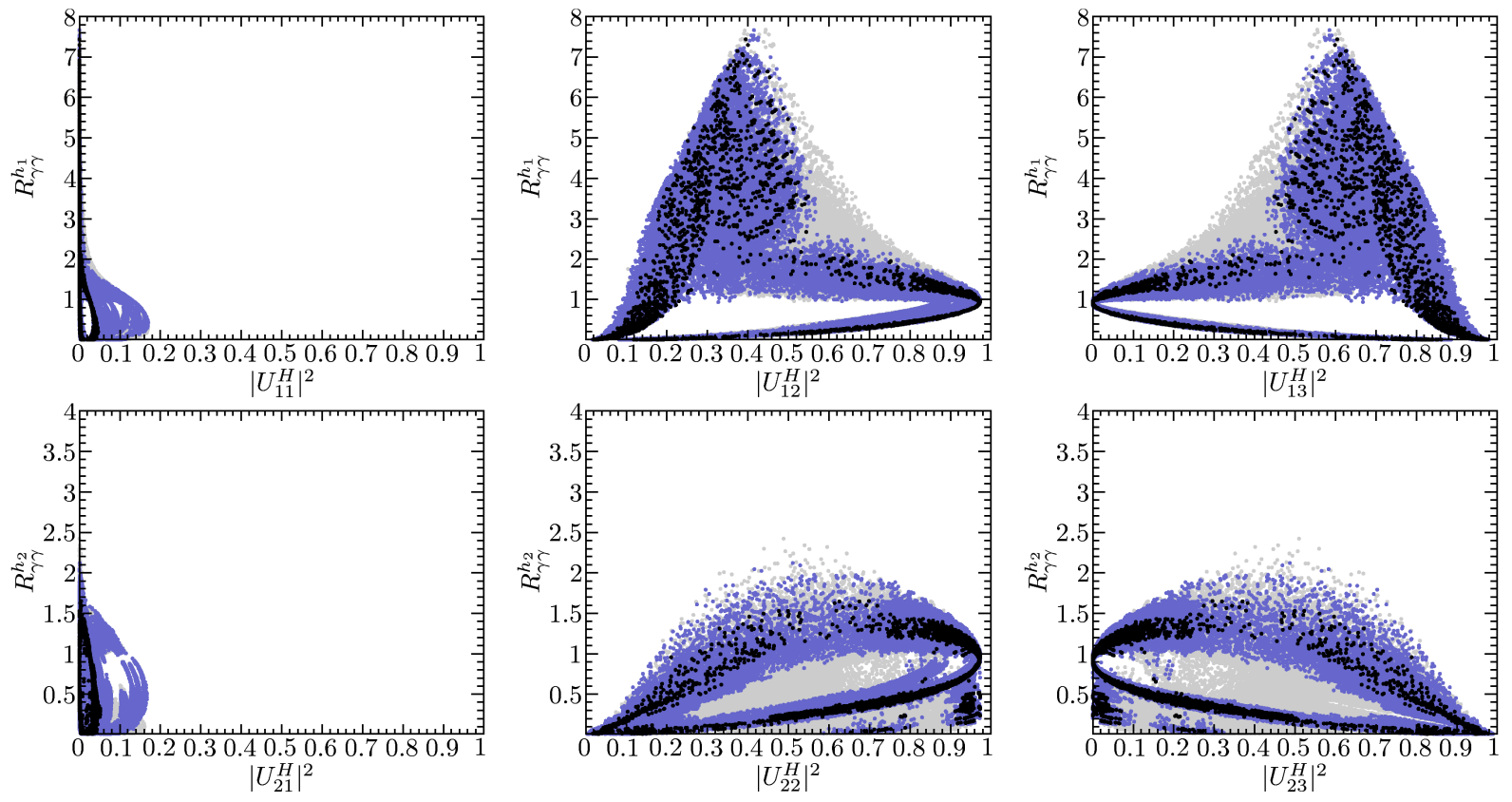

Figure 9: Results from the NMSSM parameter scan on $R_{\gamma \gamma}^{h_{i}}$ and the elements $U_{i j}^{H}$ of the $\mathcal{C P}$-even Higgs mixing matrix for $h_{1}$ (top) and $h_{2}$ (bottom). The colour coding is the same as in Fig. 7 .

well as the loop-induced processes, in the total width is essential for a realistic prediction.

The results for $R_{\gamma \gamma}^{h_{1}}$ and $R_{\gamma \gamma}^{h_{2}}$ from the scan over the NMSSM parameter space are shown in Fig. 8. As before, we show the plots both on a logarithmic and a linear scale, and include the latest results on this channel taken from [1]. Looking first at $h_{1}$, the figure shows that a sizable enhancement over the SM rate is possible over the whole mass range from $m_{h_{1}}=80 \mathrm{GeV}$ to $m_{h_{1}}=130 \mathrm{GeV}$. For the range of Higgs masses below the SM limit, $m_{h_{1}}<114.4 \mathrm{GeV}$, points with a significant enhancement $R_{\gamma \gamma}^{h_{1}} \gtrsim 7$ are observed, in accordance with the results of [77] (see also [68]).

Turning to $h_{2}$, the results for $R_{\gamma \gamma}^{h_{2}}$ are similar to those for $R_{\gamma \gamma}^{h_{1}}$ in the common mass range; the observed maximal enhancement is $R_{\gamma \gamma}^{h_{2}} \gtrsim 2$ for $m_{h_{2}}$ in the range from $120 \mathrm{GeV}$ to $125 \mathrm{GeV}$. A smaller enhancement over the SM is possible for all $m_{h_{2}}<145 \mathrm{GeV}$. As $m_{h_{2}}$ approaches $160 \mathrm{GeV}$, where the on-shell decay $h_{2} \rightarrow W W^{(*)}$ opens, the rate drops to $R_{\gamma \gamma}^{h_{2}}<1$.

Comparing the results for $R_{\gamma \gamma}^{h_{i}}$ to the limits most recent limits from ATLAS (solid red) and CMS (dashed red) [1] (where the $\mathrm{LHC}_{2011}$ limits are shown in green), it is clearly visible that the NMSSM (similarly to the MSSM) can produce points with a large suppression of $R_{\gamma \gamma}$. Concerning the more interesting case $R_{\gamma \gamma}^{h_{i}}>1$, we see that the NMSSM can produce an enhancement compatible with an excess over the SM rate for Higgs production in the mass region around $125 \mathrm{GeV}$. The results of Fig. 8 show that such observed excess over the SM rate for Higgs production in the $\gamma \gamma$ channel is well compatible with both $h_{1}$ or $h_{2}$ production in the NMSSM.

In order to identify the conditions under which a significant NMSSM enhancement of $R_{\gamma \gamma}^{h_{i}}$ is possible - as explained above, this is a genuine NMSSM effect that goes beyond the 
mechanisms discussed above for the MSSM — we show in Fig. 9 the dependence of $R_{\gamma \gamma}^{h_{i}}$ on the composition of $h_{i}$ as defined in Eq. (12). Fig. 9 shows that an important requirement for $R_{\gamma \gamma}^{h_{i}}>1$ is that $U_{i 1}^{H} \simeq 0$, which means that the corresponding Higgs mass eigenstate lacks a $H_{d}$ component. In the limit $U_{i 1}^{H} \rightarrow 0$ (corresponding to $\alpha_{\text {eff }} \rightarrow 0$ in the MSSM) the otherwise dominant decay channels $h_{i} \rightarrow b \bar{b}$ and $h_{i} \rightarrow \tau \tau$ vanish (in the effective coupling approximation), thereby increasing $\mathrm{BR}\left(h_{i} \rightarrow \gamma \gamma\right)$. Since $U^{H}$ is unitary, the general sum rule

$$
\sum_{j}\left|U_{i j}^{H}\right|^{2}=1
$$

implies that points with $U_{i 1}^{H}=0$ must have $\left|U_{i 2}^{H}\right|^{2}+\left|U_{i 3}^{H}\right|^{2}=1$. From Fig. 9 it can be seen that a configuration that maximises $R_{\gamma \gamma}^{h_{i}}$ would be

$$
\left|U_{i 1}^{H}\right|^{2}=0, \quad\left|U_{i 2}^{H}\right|^{2} \simeq 0.4, \quad\left|U_{i 3}^{H}\right|^{2} \simeq 0.6 .
$$

Unlike the case of Higgs doublet mixing resulting in a small $\alpha_{\text {eff }}$ in the MSSM (which requires a low value for $M_{A}$ and a high $\mu$ ), in the NMSSM this feature is caused by a sizable singlet component of $h_{i}$. The observed $R_{\gamma \gamma}^{h_{i}}$ enhancement is therefore a genuine feature of the NMSSM which is still present even in the SM+singlet limit. On the other hand, in the MSSM limit (where $\left|U_{i 3}^{H}\right|^{2}=0$ ) points from our scan show only very small $R_{\gamma \gamma}^{h_{i}}$ enhancements. This is a consequence of the fact that we have restricted our scan in the NMSSM to large $M_{H^{ \pm}}$, large slepton and squark masses, as well as to relatively small values of $\tan \beta$ and $\mu_{\text {eff }}$, which corresponds to a parameter region in the MSSM that is complementarity to the one used for our MSSM scan.

As for the MSSM case, also in the NMSSM the decay $h_{i} \rightarrow \gamma \gamma$ is usually dominated by contributions from loops containing $W$ bosons, and we expect a corresponding correlation of $R_{\gamma \gamma}^{h_{i}}$ with the (off-shell) decays $h_{i} \rightarrow W W^{(*)}$ and $h_{i} \rightarrow Z Z^{(*)}$. This is studied in Fig. 10, where we give results for the tree-level decays $h_{1,2} \rightarrow W W^{(*)}$ using the same colour coding as above. As expected, a sizable enhancement is possible for $R_{W W}^{h_{i}}$, in particular for $h_{1}$, and a strong positive correlation between $R_{W W}^{h_{i}}$ and $R_{\gamma \gamma}^{h_{i}}$ is visible. The possibility of a simultaneous enhancement of these two modes can again be understood as an effect of the large suppression of the main fermionic coupling $h_{i} b \bar{b}$, which leads to an enhancement of the respective branching ratios. As in the case of the MSSM, despite the positive correlation between $R_{W W}^{h_{i}}$ and $R_{\gamma \gamma}^{h_{i}}$ it is nevertheless possible to have a both a slight enhancement of $R_{\gamma \gamma}^{h_{i}}$ and a slight suppression of $R_{W W}^{h_{i}}$.

In Fig. 10] we also display the ATLAS [52] and CMS [53] limits on the $H \rightarrow W W$ channel based on $\mathrm{LHC}_{2011}$. For the same reasons as in the MSSM case we do not include the new results from [1]. The $\mathrm{LHC}_{2011}$ limits exclude a SM-like Higgs for $M_{H} \gtrsim 130 \mathrm{GeV}$ (which would not change by the inclusion of the data from [1]). The points excluded by this mode can be seen as a grey strip for $m_{h_{2}} \gtrsim 130 \mathrm{GeV}$ in this figure and also in Fig. 8. For the few points above the green lines which are not excluded by $H \rightarrow W W$, another channel has a higher expected sensitivity. For $m_{h_{i}}<130 \mathrm{GeV}$ the existing limits on $h_{i} \rightarrow W W$ do not pose any constraint on the NMSSM, but an improved exclusion of the $W W$ mode at lower Higgs masses could have severe implications for the scenarios we consider. The resulting limits on $R_{\gamma \gamma}^{h_{i}}$ are competing with those from direct searches in this channel, but appear somewhat relaxed in view of the excess over the SM prediction in the $\gamma \gamma$ channel as reported in [1]. 

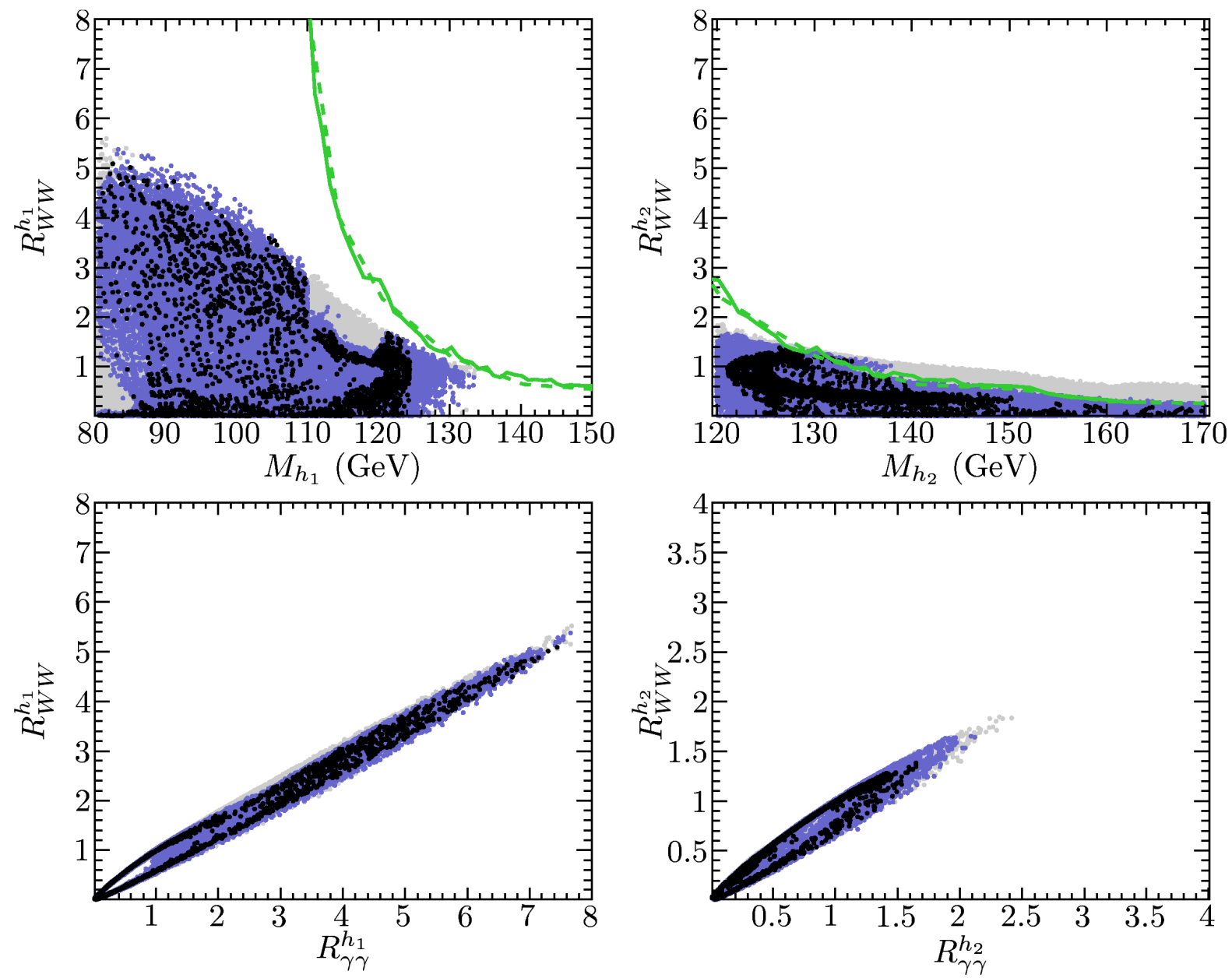

Figure 10: Results from the NMSSM parameter scan on the ratios $R_{W W}^{h_{i}}(i=1,2)$ for the NMSSM Higgs bosons $h_{1}$ (left column) and $h_{2}$ (right column). The plots in the upper row show $R_{W W}^{h_{i}}$ as a function of the respective Higgs mass. The 95\% CL exclusion limits for the $W W^{(*)}$ channel from $\mathrm{LHC}_{2011}$ data from ATLAS [52] (solid line) and CMS [53] (dashed line) are also shown. The plots in the lower row show the correlation of $R_{W W}^{h_{i}}$ and $R_{\gamma \gamma}^{h_{i}}$. The colour coding is the same as in Fig. [7.

The combination of those two channels offers an excellent opportunity for cross-checks and model testing.

\section{3 $\mathcal{C P}$-odd Higgs decays in the NMSSM and the MSSM}

For completeness, we have also investigated decays of $\mathcal{C P}$-odd Higgs bosons in the MSSM and in the NMSSM. In the following we denote a generic $\mathcal{C P}$-odd Higgs boson as $\phi_{A}$. As a consequence of gauge invariance, the $\gamma \phi_{A} Z$ vertex vanishes in both models at the tree-level. The vertices $V V \phi_{A}(V=\gamma, g, Z, W)$ vanish by virtue of $\mathcal{C P}$-invariance [78]. Accordingly, all these vertices only appear at the one-loop level.

The MSSM contribution to $A \rightarrow \gamma \gamma, g g$ has been evaluated by many groups (see [18] for a comprehensive list of references). The MSSM prediction for $A \rightarrow W W$ was first studied 
in Refs. [79, 80, however only heavy SM fermions were included. In Ref. [80], chargino and neutralino contributions to $A \rightarrow W W$ were included together with the SM fermions. In the MSSM the BR's of $A \rightarrow \gamma \gamma$ and $A \rightarrow W W$ are of $\mathcal{O}\left(10^{-4}\right)$ and $\mathcal{O}\left(10^{-3}\right)$, respectively, for small $\tan \beta$ and decrease for large $\tan \beta$. Consequently, for a production cross section similar to a SM Higgs boson with the same mass, $R_{\gamma \gamma}^{A}$ can be order of $\mathcal{O}\left(10^{-2}\right)$. The prospects for the observation of those modes at the LHC are not very encouraging.

In order to assess the NMSSM potential for the observation of the $\mathcal{C P}$-odd Higgs bosons in these loop-induced decay channels, a random scan over the six-dimensional parameter space of the Higgs sector has been performed (see Tab. 3). In this scan the lightest $\mathcal{C} \mathcal{P}$ odd Higgs boson has a mass ranging from about 80 to $250 \mathrm{GeV}$. Due to the mixing of doublet and singlet states, Eq. (13), the NMSSM $\mathcal{C} \mathcal{P}$-odd Higgs bosons can have couplings to fermions that are significantly smaller than in the MSSM. Contrary to our analysis in the previous section where we compared to the SM, we now compare the NMSSM results for the light $\mathcal{C P}$-odd Higgs boson $a_{1}$ to the results for the $\mathcal{C P}$-odd Higgs boson in the MSSM (with $\left.m_{a_{1}}=M_{A}\right)$. Both in the MSSM and the NMSSM, we compute the total decay widths of $\mathcal{C P}$-odd Higgs according to

$$
\Gamma_{\text {tot }}\left(\phi_{A}\right)=\frac{1}{m_{\phi_{A}}} \operatorname{Im}\left[\Sigma_{\phi_{A}}\left(m_{\phi_{A}}^{2}\right)\right]+\sum_{V=\gamma, g, Z, W} \Gamma\left(\phi_{A} \rightarrow V V\right) .
$$

In order to compare the NMSSM to the MSSM we define the ratio of di-photon cross-section normalised to the MSSM rate as follows

$$
\hat{R}_{\gamma \gamma}^{a_{j}}=\frac{\left[\sigma\left(g g \rightarrow a_{j}\right) \times \operatorname{BR}\left(a_{j} \rightarrow \gamma \gamma\right)\right]_{\mathrm{NMSSM}}}{[\sigma(g g \rightarrow A) \times \operatorname{BR}(A \rightarrow \gamma \gamma)]_{\mathrm{MSSM}}} .
$$

In the case where $\lambda, \kappa \rightarrow 0$ (with $\kappa / \lambda$ held constant), which corresponds to the MSSM limit (see above), the interactions of the singlet field with the MSSM Higgs doublets vanish, i.e. the singlet field decouples. The decoupled singlet field itself has no gauge couplings. In this MSSM limit, one finds that one of the NMSSM $\mathcal{C} \mathcal{P}$-odd Higgs bosons, $a_{k}$ (which does not necessarily coincide with $a_{1}$ ) behaves like the MSSM $A$ boson. The MSSM phenomenology is fully retrieved in the Higgs sector, the total decay width of the $\mathcal{C P}$-odd Higgs in the NMSSM approaches the MSSM value, and we find $\hat{R}_{\gamma \gamma}^{a_{k}} \sim 1$. However, even in this limit, in some cases the distinction of the two models might still be possible via the neutralino sector [81].

Far from the MSSM limit, the coupling $a_{k} f \bar{f}$, which is proportional to the doublet-singlet mixing, gets suppressed and the NMSSM phenomenology can differ from the MSSM. In our scan we find an enhancement of the $\operatorname{BR}\left(a_{1} \rightarrow \gamma \gamma\right)$ of up to 100 over the MSSM. However, also the $a_{1}$ production rate gets suppressed in this case, leading to (at most) $\hat{R}_{\gamma \gamma}^{a_{1}} \sim 2$. Consequently, as for the MSSM, also $a_{1}$ is expected to remain unobservable in this channel at the LHC (and we do not show the plots for this channel). 


\section{Conclusions}

The discovery of a new particle with properties that are compatible with a Higgs boson has started a new era, with the goals to reveal whether the new particle is indeed a Higgs boson and whether its detailed phenomenology is consistent with the SM predictions or whether there is evidence for physics beyond the SM. The observations reported both by ATLAS and CMS that the new state has a signal strength in the $\gamma \gamma$ channel that is considerably higher than the one expected in the SM may be a first hint into this direction, although the statistical significance of this deviation from the SM prediction is not sufficien at present to draw a definite conclusion. In view of the possibility of an enhanced rate in the $\gamma \gamma$ channel, it is nevertheless interesting to investigate the predictions of possible alternatives to the SM in this context. We have carried out such an analysis in the present paper, comparing the predictions for Higgs boson production in gluon fusion, the main production channel at the LHC, and its subsequent decay into two photons in the SM, the MSSM, and the NMSSM. We have furthermore analysed the $W W^{*}$ channel, which is strongly correlated with the $\gamma \gamma$ mode.

While for the predictions in the SM and the MSSM we have used the well-known code FeynHiggs, for the predictions in the NMSSM we have developed a new framework consisting in particular of an appropriate model file for the program FeynArts and the input on masses, mixing angles, etc. needed for the numerical evaluation. In this set-up we can make use of the highly automated programs FormCalc and LoopTools for the evaluation of the relevant observables. In the present implementation NMSSMTools is used for higher-order corrections to the masses of the external Higgs bosons. Numerous tests have been performed to verify the implementation; among other things we evaluated more than 150 loop-induced processes in the NMSSM and checked the results for UV-finiteness.

We have presented results for Higgs-boson production in gluon fusion and its decays into $\gamma \gamma$ and $W W^{*}$ within the MSSM and the NMSSM, normalised to the SM prediction. We have analysed in detail possible mechanisms for the enhancement (but also the suppression) of those channels in both models. In this context we have investigated in particular whether an enhancement of the $\gamma \gamma$ rate for a Higgs mass of about $125 \mathrm{GeV}$ is compatible with existing limits on the parameter space arising from theoretical constraints as well as from the limits from direct searches for supersymmetric particles, from the Higgs searches at LEP, the Tevatron and the LHC (where we have incorporated the limits based on the data presented in 2011), from electroweak precision observables and from flavour physics. Performing parameter scans in both models, we have then confronted the points passing all the above constraints with the latest results of the Higgs searches in the $\gamma \gamma$ channel that have just been announced by ATLAS and CMS. We have found that an enhanced rate of Higgs production and decay to two photons can easily be realised in both models, the MSSM as well as in the NMSSM. This holds not only for the lightest $\mathcal{C} \mathcal{P}$-even Higgs boson in the models, but also for the second lightest $\mathcal{C} \mathcal{P}$-even Higgs boson in both the MSSM and the NMSSM. In this latter interpretation in both models the lightest $\mathcal{C P}$-even Higgs boson possesses a strongly suppressed coupling to gauge bosons and escapes all existing direct searches.

Within the MSSM we have analysed the mechanisms that can lead to such a enhanced $\gamma \gamma$ rate in comparison to the SM prediction. Besides the presence of light scalar taus, in particular a suppression of the $b \bar{b}$ decay mode results in an enhanced $\gamma \gamma$ rate. This 
suppression can either be caused by Higgs-boson propagator corrections entering the effective mixing angle, or by the so-called $\Delta_{b}$ corrections.

Within the NMSSM the above mentioned mechanisms can also naturally be realised, and we focused on additional mechanisms that are genuine for the NMSSM. We found that in particular the doublet-singlet mixing can result in a substantial suppression of the $b \bar{b}$ mode, resulting again in the desired enhancement in the $\gamma \gamma$ rate with respect to the SM prediction.

Finally we have briefly analysed the decays of $\mathcal{C} \mathcal{P}$-odd Higgs bosons to two photons. While an enhancement with respect to the corresponding rate in the MSSM is possible in the NMSSM, a signal such as reported by ATLAS and CMS [1] cannot be accounted for by a $\mathcal{C P}$-odd Higgs.

\section{Acknowledgements}

We thank C. Duhr, B. Fuks, S. Liebler and F. Staub for helpful discussions. We are also grateful to T. Stefaniak and O. Brein for discussions and assistance with HiggsBounds. The work of S.H. was partially supported by CICYT (grant FPA 2007-66387 and FPA 2010 22163-C02-01), and by the Spanish MICINN's Consolider-Ingenio 2010 Program under grant MultiDark CSD2009-00064. Work supported in part by the European Community's MarieCurie Research Training Network under contract MRTN-CT-2006-035505 "Tools and Precision Calculations for Physics Discoveries at Colliders" and by the Collaborative Research Center SFB676 of the DFG, "Particles, Strings, and the Early Universe".

\section{References}

[1] See: https://indico.cern.ch/conferenceDisplay ·py?conf Id=197461 .

[2] G. Aad et al. (ATLAS Collaboration), Phys. Lett. B 710, 49 (2012) [arXiv:1202.1408 [hep-ex]].

[3] S. Chatrchyan et al. (CMS Collaboration), Phys. Lett. B 710, 26 (2012) [arXiv:1202.1488 [hep-ex]].

[4] CMS Collaboration, [arXiv:1202.1488 [hep-ex]].

[5] CDF Collaboration, DØCollaboration, [arXiv:1207.0449 [hep-ex]].

[6] H.P. Nilles, Phys. Rept. 110 (1984) 1; H.E. Haber and G.L. Kane, Phys. Rept. 117 (1985) 75; R. Barbieri, Riv. Nuovo Cim. 11 (1988)1.

[7] U. Ellwanger, C. Hugonie and A. M. Teixeira, Phys. Rept. 496 (2010) 1 [arXiv:0910.1785 [hep-ph]]; M. Maniatis, Int. J. Mod. Phys. A 25 (2010) 3505 [arXiv:0906.0777 [hep-ph]].

[8] J. Ellis, J. F. Gunion, H. A. Haber, L. Roszkowski and F. Zwirner, Phys. Rev. D. 39 (1989) 844.

[9] D. J. Miller, R. Nevzorov and P. M. Zerwas, Nucl. Phys. B 681 (2004) 3 [arXiv:hepph/0304049]. 
[10] A. Djouadi et al., JHEP 0807 (2008) 002 [arXiv:0801.4321 [hep-ph]].

[11] U. Ellwanger, J. F. Gunion and C. Hugonie, [arXiv:hep-ph/0111179]; U. Ellwanger, J. F. Gunion, C. Hugonie and S. Moretti, [arXiv:hep-ph/0305109]; U. Ellwanger, J. F. Gunion, C. Hugonie and S. Moretti, [arXiv:hep-ph/0401228]; S. Moretti, S. Munir and P. Poulose, Phys. Lett. B 644 (2007) 241 [arXiv:hep-ph/0608233]; J. R. Forshaw, J. F. Gunion, L. Hodgkinson, A. Papaefstathiou and A. D. Pilkington, JHEP 0804 (2008) 090 [arXiv:0712.3510 [hep-ph]]; A. Belyaev, S. Hesselbach, S. Lehti, S. Moretti, A. Nikitenko and C. H. Shepherd-Themistocleous, [arXiv:0805.3505 [hep-ph]].

[12] K. E. Williams and G. Weiglein, Phys. Lett. B 660 (2008) 217 [arXiv:0710.5320 [hepph]]; K. E. Williams, H. Rzehak and G. Weiglein, Eur. Phys. J. C 71 (2011) 1669 [arXiv:1103.1335 [hep-ph]].

[13] R. Dermisek and J. F. Gunion, Phys. Rev. Lett. 95 (2005) 041801 [arXiv:hepph/0502105]; R. Dermisek and J. F. Gunion, Phys. Rev. D 73 (2006) 111701 [arXiv:hep-ph/0510322]; R. Dermisek and J. F. Gunion, Phys. Rev. D 75 (2007) 075019 [arXiv:hep-ph/0611142]; R. Dermisek and J. F. Gunion, Phys. Rev. D 76 (2007) 095006 [arXiv:0705.4387 [hep-ph]]; R. Dermisek and J. F. Gunion, Phys. Rev. D 79 (2009) 055014 [arXiv:0811.3537 [hep-ph]]; R. Dermisek and J. F. Gunion, Phys. Rev. D 81 (2010) 075003 [arXiv:1002.1971 [hep-ph]].

[14] O. Stal and G. Weiglein, JHEP 1201 (2012) 071 [arXiv:1108.0595 [hep-ph]].

[15] U. Ellwanger, Eur. Phys. J. C 71 (2011) 1782 [arXiv:1108.0157 [hep-ph]].

[16] P. Draper, T. Liu, C. E. M. Wagner, L. -T. Wang and H. Zhang, Phys. Rev. Lett. 106 (2011) 121805 [arXiv:1009.3963 [hep-ph]]; D. A. Vasquez, G. Belanger, C. Boehm, A. Pukhov and J. Silk, Phys. Rev. D 82 (2010) 115027 [arXiv:1009.4380 [hep-ph]].

[17] M. Frank, T. Hahn, S. Heinemeyer, W. Hollik, R. Rzehak and G. Weiglein, JHEP 02 047 (2007) [arXiv:hep-ph/0611326].

[18] A. Djouadi, Phys. Rept. 459 (2008) 1 [arXiv:hep-ph/0503173].

[19] S. Heinemeyer, Int. J. Mod. Phys. A 21 (2006) 2659 [arXiv:hep-ph/0407244]; S. Heinemeyer, W. Hollik and G. Weiglein, Phys. Rept. 425 (2006) 265 [hep-ph/0412214].

[20] U. Ellwanger, Phys. Lett. B 303 (1993) 271 [arXiv:hep-ph/9302224]; T. Elliott, S. F. King and P. L. White, Phys. Lett. B 305 (1993) 71 [arXiv:hep-ph/9302202]; T. Elliott, S. F. King and P. L. White, Phys. Lett. B 314 (1993) 56 [arXiv:hepph/9305282]; T. Elliott, S. F. King and P. L. White, Phys. Rev. D 49 (1994) 2435 [arXiv:hep-ph/9308309]; P. N. Pandita, Phys. Lett. B 318 (1993) 338; U. Ellwanger and C. Hugonie, Phys. Lett. B 623 (2005) 93 [arXiv:hep-ph/0504269]; G. Degrassi and P. Slavich, Nucl. Phys. B 825 (2010) 119 [arXiv:0907.4682 [hep-ph]]; F. Staub, W. Porod and B. Herrmann, JHEP 1010 (2010) 040 [arXiv:1007.4049 [hep-ph]].

[21] R. Benbrik, M. Gomez Bock, S. Heinemeyer, O. Stål, G. Weiglein and L. Zeune, work in progress. 
[22] S. Heinemeyer, W. Hollik and G. Weiglein, Comput. Phys. Commun. 124 (2000) 76 [arXiv:hep-ph/9812320]; T. Hahn, S. Heinemeyer, W. Hollik, H. Rzehak and G. Weiglein, Comput. Phys. Commun. 180 (2009) 1426; see www.feynhiggs.de.

[23] S. Heinemeyer, W. Hollik and G. Weiglein, Eur. Phys. J. C 9 (1999) 343 [arXiv:hep$\mathrm{ph} / 9812472]$.

[24] G. Degrassi, S. Heinemeyer, W. Hollik, P. Slavich and G. Weiglein, Eur. Phys. J. C 28 (2003) 133 [arXiv:hep-ph/0212020].

[25] U. Ellwanger, J. F. Gunion and C. Hugonie, JHEP 0502 (2005) 066 [arXiv:hepph/0406215]; U. Ellwanger and C. Hugonie, Comput. Phys. Commun. 175 (2006) 290 [arXiv:hep-ph/0508022]; G. Belanger, F. Boudjema, C. Hugonie, A. Pukhov and A. Semenov, JCAP 0509 (2005) 001 [arXiv:hep-ph/0505142].

[26] D. Das, U. Ellwanger and A. M. Teixeira, Comput. Phys. Commun. 183 (2012) 774 [arXiv:1106.5633 [hep-ph]].

[27] W. Porod, Comput. Phys. Commun. 153 (2003) 275 [arXiv:hep-ph/0301101]; W. Porod and F. Staub, [arXiv:1104.1573 [hep-ph]].

[28] F. Staub, [arXiv:0806.0538 [hep-ph]]; F. Staub, Comput. Phys. Commun. 181 (2010) 1077 [arXiv:0909.2863 [hep-ph]].

[29] K. Ender, T. Graf, M. Mühlleitner and H. Rzehak, [arXiv:1111.4952 [hep-ph]]; T. Graf, R. Grober, M. Mühlleitner, H. Rzehak and K. Walz, arXiv:1206.6806 [hep-ph].

[30] S. Heinemeyer, O. Stål and G. Weiglein, Phys. Lett. B 710 (2012) 201 [arXiv:1112.3026 [hep-ph]];

[31] M. Carena, S. Gori, N. R. Shah and C. E. M. Wagner, JHEP 1203 (2012) 014 [arXiv:1112.3336 [hep-ph]]; M. Carena, S. Gori, N. R. Shah, C. E. M. Wagner and L. -T. Wang, [arXiv:1205.5842 [hep-ph]].

[32] H. Baer, V. Barger and A. Mustafayev, [arXiv:1112.3017 [hep-ph]]; A. Arbey, M. Battaglia, A. Djouadi, F. Mahmoudi and J. Quevillon, Phys. Lett. B 708 (2012) 162 [arXiv:1112.3028 [hep-ph]]; M. Kadastik, K. Kannike, A. Racioppi and M. Raidal, JHEP 1205 (2012) 061 [arXiv:1112.3647 [hep-ph]]; J. Cao, Z. Heng, D. Li and J. M. Yang, Phys. Lett. B 710 (2012) 665 [arXiv:1112.4391 [hep-ph]]; F. Brümmer and W. Buchmüller, JHEP 1205 (2012) 006 [arXiv:1201.4338 [hep-ph]]; D. Carmi, A. Falkowski, E. Kuflik and T. Volansky, arXiv:1202.3144 [hep-ph]; J. Ellis and K. A. Olive, arXiv:1202.3262 [hep-ph]; N. Desai, B. Mukhopadhyaya and S. Niyogi, arXiv:1202.5190 [hep-ph]; T. Cheng, J. Li, T. Li, D. V. Nanopoulos and C. Tong, arXiv:1202.6088 [hep-ph]; M. Asano, S. Matsumoto, M. Senami and H. Sugiyama, arXiv:1202.6318 [hep-ph]; J. Cao, Z. Heng, J. M. Yang and J. Zhu, arXiv:1203.0694 [hep-ph]; A. Choudhury and A. Datta, JHEP 1206 (2012) 006 [arXiv:1203.4106 [hepph]]; P. P. Giardino, K. Kannike, M. Raidal and A. Strumia, arXiv:1203.4254 [hepph]; M. A. Ajaib, I. Gogoladze, F. Nasir and Q. Shafi, arXiv:1204.2856 [hep-ph]; 
F. Brümmer, S. Kraml and S. Kulkarni, arXiv:1204.5977 [hep-ph]; J. L. Evans, M. Ibe and T. T. Yanagida, arXiv:1204.6085 [hep-ph]. A. Fowlie, M. Kazana, K. Kowalska, S. Munir, L. Roszkowski, E. M. Sessolo, S. Trojanowski and Y. -L. S. Tsai, arXiv:1206.0264 [hep-ph].

[33] U. Ellwanger, JHEP 1203 (2012) 044 [arXiv:1112.3548 [hep-ph]].

[34] L. J. Hall, D. Pinner and J. T. Ruderman, [arXiv:1112.2703 [hep-ph]]; A. Arvanitaki and G. Villadoro, [arXiv:1112.4835 [hep-ph]]; J. F. Gunion, Y. Jiang and S. Kraml, [arXiv:1201.0982 [hep-ph]]; S. F. King, M. Mühlleitner and R. Nevzorov, Nucl. Phys. B 860 (2012) 207 [arXiv:1201.2671 [hep-ph]]; D. A. Vasquez, G. Belanger, C. Boehm, J. Da Silva, P. Richardson and C. Wymant, arXiv:1203.3446 [hep-ph]; U. Ellwanger and C. Hugonie, arXiv:1203.5048 [hep-ph]; E. Gabrielli, K. Kannike, B. Mele, A. Racioppi and M. Raidal, arXiv:1204.0080 [hep-ph]; J. Rathsman and T. Rossler, arXiv:1206.1470 [hep-ph].

[35] J. Cao, Z. Heng, J. M. Yang, Y. Zhang and J. Zhu, JHEP 1203 (2012) 086 [arXiv:1202.5821 [hep-ph]].

[36] J. Kublbeck, M. Bohm and A. Denner, Comput. Phys. Commun. 60 (1990) 165;

A. Denner, H. Eck, O. Hahn and J. Kublbeck, Phys. Lett. B 291 (1992) 278;

A. Denner, H. Eck, O. Hahn and J. Kublbeck, Nucl. Phys. B 387 (1992) 467;

J. Kublbeck, H. Eck and R. Mertig, Nucl. Phys. Proc. Suppl. 29A (1992) 204;

T. Hahn, Comput. Phys. Commun. 140 (2001) 418 [hep-ph/0012260];

T. Hahn and C. Schappacher, Comput. Phys. Commun. 143 (2002) 54 [hep$\mathrm{ph} / 0105349]$.

[37] T. Hahn and M. Perez-Victoria, Comput. Phys. Commun. 118 (1999) 153 [hep$\mathrm{ph} / 9807565]$.

[38] T. Hahn, Comput. Phys. Commun. 178 (2008) 217 [hep-ph/0611273].

[39] See also: G. J. van Oldenborgh, Comput. Phys. Commun. 66 (1991) 1.

[40] J. A. M. Vermaseren, math-ph/0010025.

[41] N. D. Christensen and C. Duhr, Comput. Phys. Commun. 180 (2009) 1614 [arXiv:0806.4194 [hep-ph]].

[42] P. Bechtle, O. Brein, S. Heinemeyer, G. Weiglein and K. E. Williams, Comput. Phys. Commun. 181 (2010) 138 [arXiv:0811.4169 [hep-ph]]; ibid. 182 (2011) 2605 [arXiv:1102.1898 [hep-ph]].

[43] U. Ellwanger, M. Rausch de Traubenberg and C. A. Savoy, Nucl. Phys. B 492 (1997) 21 [arXiv:hep-ph/9611251].

[44] S. F. King and P. L. White, Phys. Rev. D 52 (1995) 4183 [arXiv:hep-ph/9505326]; M. Masip, R. Munoz-Tapia and A. Pomarol, Phys. Rev. D 57 (1998) R5340 [arXiv:hepph/9801437]. 
[45] LEP Higgs working group, Phys. Lett. B 565 (2003) 61 [arXiv:hep-ex/0306033].

[46] LEP Higgs working group, Eur. Phys. J. C 47 (2006) 547 [arXiv:hep-ex/0602042].

[47] F. Mahmoudi, J. Rathsman, O. Stål and L. Zeune, Eur. Phys. J. C 71 (2011) 1608 [arXiv:1012.4490 [hep-ph]].

[48] CMS Collaboration, CMS PAS HIG-11-020.

[49] CMS Collaboration, CMS-PAS-HIG-11-029.

[50] ATLAS Collaboration, ATLAS-CONF-2011-161.

[51] CMS Collaboration, CMS-HIG-11-030.

[52] ATLAS Collaboration, [arXiv:1112.2577 [hep-ex]].

[53] CMS Collaboration, CMS-HIG-11-024.

[54] K. Nakamura et al. (Particle Data Group), J. Phys. G 37 (2010) 075021.

[55] ATLAS Collaboration, ATLAS-CONF-2012-033; CMS Collaboration, CMS-PAS-SUS-11-016 .

[56] M. Davier, A. Hoecker, B. Malaescu and Z. Zhang, Eur. Phys. J. C 71 (2011) 1515 [arXiv:1010.4180 [hep-ph]].

[57] G. Bennett et al. (The Muon $g-2$ Collaboration), Phys. Rev. Lett. 92 (2004) 161802 [arXiv:hep-ex/0401008]; Phys. Rev. D 73 (2006) 072003 [arXiv:hep-ex/0602035].

[58] J. F. Gunion, D. Hooper and B. McElrath, Phys. Rev. D 73 (2006) 015011 [arXiv:hepph/0509024]; J. F. Gunion, JHEP 0908 (2009) 032 [arXiv:0808.2509 [hep-ph]].

[59] F. Domingo and U. Ellwanger, JHEP 0807 (2008) 079 [arXiv:0806.0733 [hep-ph]].

[60] G. Hiller, Phys. Rev. D 70 (2004) 034018 [arXiv:hep-ph/0404220].

[61] F. Domingo and U. Ellwanger, JHEP 0712 (2007) 090 [arXiv:0710.3714 [hep-ph]].

[62] B. A. Dobrescu, G. L. Landsberg and K. T. Matchev, Phys. Rev. D 63 (2001) 075003 [arXiv:hep-ph/0005308]; B. A. Dobrescu and K. T. Matchev, JHEP 0009 (2000) 031 [arXiv:hep-ph/0008192]; K. Cheung, J. Song and Q. S. Yan, Phys. Rev. Lett. 99 (2007) 031801 [arXiv:hep-ph/0703149]; M. A. Sanchis-Lozano, [arXiv:0709.3647 [hep-ph]].

[63] E. Barberio et al. (Heavy Flavor Averaging Group (HFAG)), [arXiv:hep-ex/0603003]. See also updated data on the HFAG webpage.

[64] CMS and LHCb Collaborations, CMS-PAS-BPH-11-019, LHCb-CONF-2011-047.

[65] D. Asner et al. (Heavy Flavor Averaging Group (HFAG)), [arXiv:1010.1589 [hep-ex]]. 
[66] A. Abulencia et al. (CDF Collaboration), Phys. Rev. Lett. 97 (2006) 242003 [arXiv:hepex/0609040].

[67] CMS Collaboration, CMS-PAS-HIG-12-001.

[68] J. Cao, Z. Heng, T. Liu and J. M. Yang, Phys. Lett. B 703 (2011) 462 [arXiv:1103.0631 [hep-ph]].

[69] N. D. Christensen, T. Han and S. Su, arXiv:1203.3207 [hep-ph];

[70] A. Arvanitaki and G. Villadoro, arXiv:1112.4835 [hep-ph].

[71] S. Heinemeyer, W. Hollik and G. Weiglein, Eur. Phys. J. C 16 (2000) 139 [arXiv:hep$\mathrm{ph} / 0003022]$.

[72] M. Carena, S. Heinemeyer, C.E.M. Wagner and G. Weiglein, [arXiv:hep-ph/0202167].

[73] R. Hempfling, Phys. Rev. D 49 (1994) 6168; L. Hall, R. Rattazzi and U. Sarid, Phys. Rev. D 50 (1994) 7048 [arXiv:hep-ph/9306309]; M. Carena, M. Olechowski, S. Pokorski and C. Wagner, Nucl. Phys. B 426 (1994) 269 [arXiv:hep-ph/9402253].

[74] M. Carena, D. Garcia, U. Nierste and C. Wagner, Nucl. Phys. B 577 (2000) 577 [arXiv:hep-ph/9912516].

[75] D. Noth and M. Spira, Phys. Rev. Lett. 101 (2008) 181801 [arXiv:0808.0087 [hep-ph]]; JHEP 1106 (2011) 084 [arXiv:1001.1935 [hep-ph]].

[76] J. Frere, D. Jones and S. Raby, Nucl. Phys. B 222 (1983) 11; M. Claudson, L. Hall and I. Hinchliffe, Nucl. Phys. B 228 (1983) 501; C. Kounnas, A. Lahanas, D. Nanopoulos and M. Quiros, Nucl. Phys. B 236 (1984) 438; J. Gunion, H. Haber and M. Sher, Nucl. Phys. B 306 (1988) 1; J. Casas, A. Lleyda and C. Munoz, Nucl. Phys. B 471 (1996) 3 [arXiv:hep-ph/9507294]; P. Langacker and N. Polonsky, Phys. Rev. D 50 (1994) 2199 [arXiv:hep-ph/9403306]; A. Strumia, Nucl. Phys. B 482 (1996) 24 [arXiv:hep$\mathrm{ph} / 9604417]$.

[77] U. Ellwanger, Phys. Lett. B 698 (2011) 293 [arXiv:1012.1201 [hep-ph]].

[78] G. Keller and D. Wyler, Nucl. Phys. B 274 (1986) 410.

[79] J. F. Gunion, H. E. Haber and C. Kao, Phys. Rev. D 46 (1992) 2907.

[80] P. H. Chankowski, S. Pokorski and J. Rosiek, Nucl. Phys. B 423 (1994) 497.

[81] G. A. Moortgat-Pick, S. Hesselbach, F. Franke and H. Fraas, JHEP 0506 (2005) 048 [arXiv:hep-ph/0502036]. 